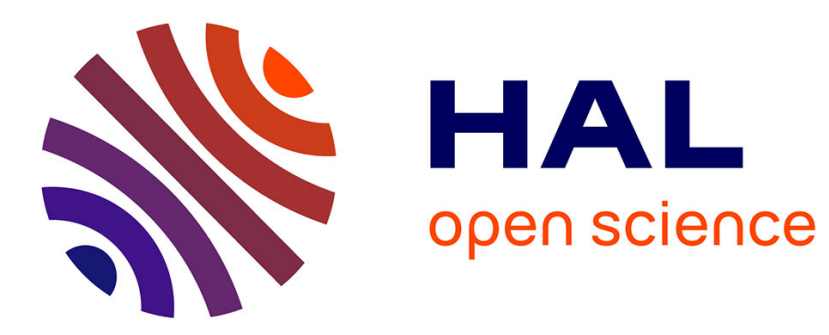

\title{
Vortex flow evolution in a growing microdroplet during co-flow in coaxial capillaries
}

\author{
S A Vagner, S A Patlazhan, C A Serra, Denis Funfschilling
}

\section{To cite this version:}

S A Vagner, S A Patlazhan, C A Serra, Denis Funfschilling. Vortex flow evolution in a growing microdroplet during co-flow in coaxial capillaries. Physics of Fluids, 2021, 10.1063/5.0057353 . hal03423110

\section{HAL Id: hal-03423110 https://hal.science/hal-03423110}

Submitted on 9 Nov 2021

HAL is a multi-disciplinary open access archive for the deposit and dissemination of scientific research documents, whether they are published or not. The documents may come from teaching and research institutions in France or abroad, or from public or private research centers.
L'archive ouverte pluridisciplinaire HAL, est destinée au dépôt et à la diffusion de documents scientifiques de niveau recherche, publiés ou non, émanant des établissements d'enseignement et de recherche français ou étrangers, des laboratoires publics ou privés. 


\title{
Vortex flow evolution in a growing microdroplet during co-flow in coaxial capillaries
}

\author{
S.A. Vagner, ${ }^{1}$ S.A. Patlazhan, ${ }^{1,2}$ C.A. Serra, ${ }^{3}$ and D. Funfschilling ${ }^{4}$ \\ 1) Institute of Problems of Chemical Physics, Russian Academy of Sciences, Academician Semenov Avenue 1, Chernogolovka, \\ Moscow Region 142432, Russia \\ 2) N.N. Semenov Federal Research Center for Chemical Physics, Russian Academy of Sciences, Kosygin Street 4, Moscow 119991, \\ Russia ${ }^{\text {a) }}$ \\ ${ }^{3)}$ Université de Strasbourg, CNRS, Institut Charles Sadron UPR 22, F-67000 Strasbourg, \\ France \\ ${ }^{4)}$ Université de Strasbourg, CNRS, ICube UMR 7357, 2, rue Boussingault, F-67000 Strasbourg, \\ France
}

(Dated: 24 June 2021)

Using Micro-Particle Image Velocimetry ( $\mu$ PIV), the convective flow inside a silicone oil droplet was investigated in details during its formation in coaxial capillaries under co-flow in a water/glycerol mixture continuous phase. The analysis of $\mu \mathrm{PIV}$ measured flow field revealed that two characteristic flow areas exist in the droplet in formation: an inflow zone and a circulation zone. The intensity of vortex flow in these zones was estimated by calculating the average angular velocity of these vortices under the condition of no shear for different dispersed phase and continuous phase flow rates and for different viscosity ratios between the two phases. The evolution of the vortex flow pattern inside the droplet was investigated thoroughly all the way from the step of their formation to the step of free-moving droplet. The results of this study are important for understanding the mixing processes inside the droplet at different stages of its formation.

\section{INTRODUCTION}

The dynamic behavior of droplets under various flow conditions has attracted the attention of researchers for many years, since the classical works of Rayleigh ${ }^{1}$ and Taylor ${ }^{2}$. A renewed interest in these studies arose with the development of microfluidic technologies aiming at producing monodisperse microdroplets. The excellent optical access that they provide made it possible to study the formation of droplet under confinement ${ }^{3-14}$, as well as to use new experimental techniques, such as Micro-Particle Image Velocimetry $(\mu \mathrm{PIV})^{15-23}$. The dynamic response of microdroplets is controlled by a set of parameters such as flow rates, capillary forces, rheological properties of dispersed and continuous phases, etc. Due to the high surface to volume ratio, heat transfer between microdroplets and the continuous phase is quite efficient. For the same reason of high surface-to-volume ratio, the heat transfer between the continuous phase and the microsystem is also very good, which results in an excellent control of the temperature in these systems. Using a microdroplet as a microreactor leads to a number of advantages over a microchannel filled with the reacting media ${ }^{24-30}$. On the foremost is the faster mixing of liquids achieved inside the microdroplet compared to the sluggish diffusion of species in the microchannel. Hence, microdroplets were used in chemical and biological analysis $^{31-34}$, synthesis of solid microparticles ${ }^{35-37}$, cytometry ${ }^{38-44}$, screening disease, etc. Such a wide diversity of applications for microdroplets poses new challenges, which imply that the peculiarities of fluid flow in a microdroplet have to be well known. Solving for these problems requires first to understand the dynamics of flow pattern in a microdroplet during

\footnotetext{
a) Electronic mail: sapat@yandex.ru
}

formation. It should be noted that various applications require different flow patterns within the droplet. For instance, an intense vortex flow within a microdroplet can accelerate chemical reactions due to the efficient mixing of reagents. On the other hand, this flow character could destroy some biological objects like cells. Therefore, the study of the flow pattern in a microdroplet under different flow conditions is an important challenge $^{45}$.

With regard to microfluidics, the peculiarities of fluid motion in a microdroplet when flowing in microchannels of different shapes as well as during its formation in various devices are of special interest. Several papers are focused on the study of fluid motion in a microdroplet while flowing in a rectangular microchannel. It was shown that in such channels a rather complex vortex flow can be generated in a microdroplet, which structure depends on capillary number $C a=\eta_{c} U_{c} / \sigma$, where $\eta_{c}$ and $U_{c}$ are respectively the viscosity and the average velocity of the continuous phase, and $\sigma$ the interfacial tension ${ }^{46-52}$. In particular, for a slightly stretched droplet with a relative length of $\beta=A / w=1.4$ ( $A$ is the largest droplet size and $w$ the cross section dimension of the microchannel) two critical capillary numbers, separating the flow structure into three types were identified ${ }^{48}$ : (i) a pair of elongated counterrotating vortices is formed near the side walls of the rectangular channel at small capillary numbers, as well as two pairs of counterrotating vortices in the central droplet crossection, (ii) a small increase in capillary number leads to only two outer vortices, and (iii) a further increase in capillary number results in reduction of the last vortices shifting them towards the channel axis. The flow pattern of longer droplets $(\beta>2)$ slightly changes ${ }^{47}$. If a similar symmetric pair of vortices is observed at small capillary numbers, increasing the capillary number leads to the formation of an additional pair of vortices at the caps of a droplet, i.e. the twovortex flow structure transforms into a system of six vortices. 


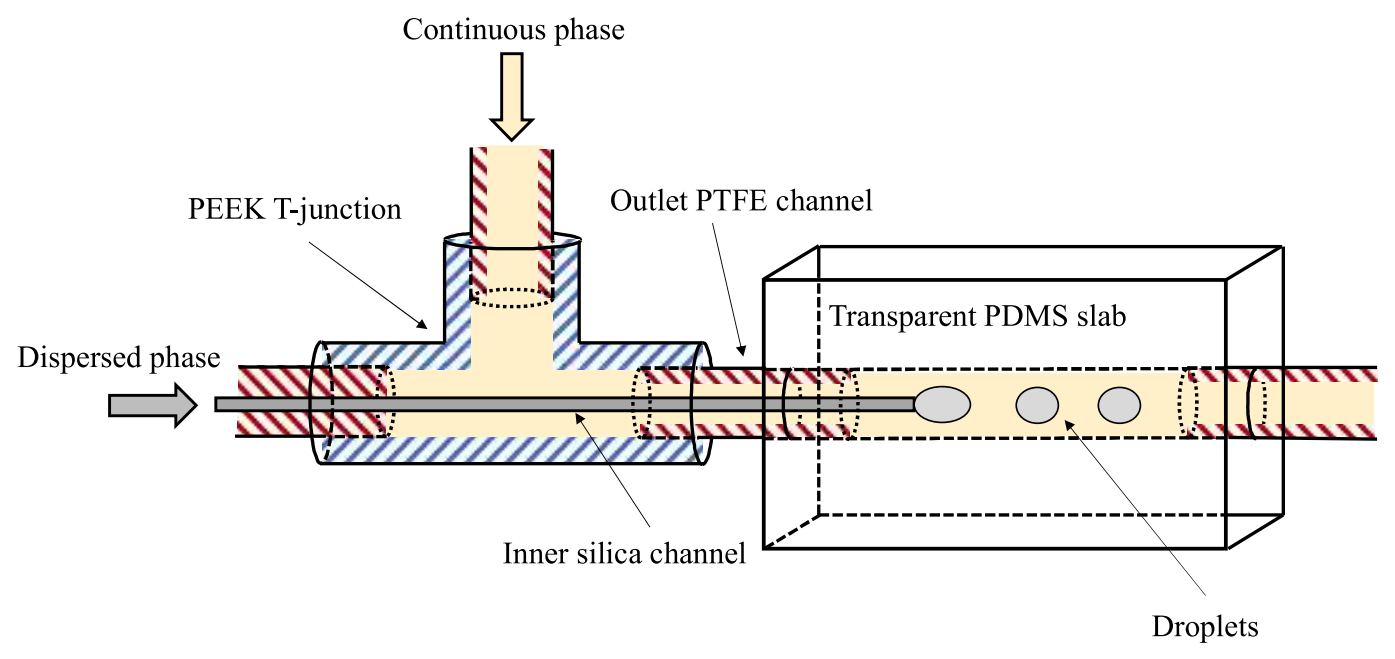

FIG. 1. Schematic drawing of the co -flow microdevice.

The critical capillary number corresponding to this process depends on the viscosity ratio of the fluid components. Notice that each pair of counterrotative symmetric vortices in the two-dimensional mid-plane of a droplet reflects the existence of a three-dimensional toroidal vortex.

When a droplet moves along a curved microchannel, the vortex pattern inside the droplet becomes more complicated. This is due to the so-called Dean vortices, which are formed due to the inertial forces ${ }^{53}$ and affect significantly the droplet shape and flow topology in its whole volume ${ }^{54-56}$. In particular, the vortex structure becomes considerably asymmetric.

Of particular interest is the study of the evolution of the vortex structure within a microdroplet during its formation in different geometries i.e., in a T-shaped capillary, flowfocusing or co-flow microchannels, in which the flow patterns are significantly different. In the work of ${ }^{57}$, the flow field in a microdrop during its formation in a flow-focusing junction was studied by $\mu$ PIV measurements at fixed ratios $q_{d}=Q_{d} / Q_{c}=0.2$ and $\eta_{d} / \eta_{c}=0.2$ of flow rates $Q_{d}$ and $Q_{c}$ and viscosities $\eta_{d}$ and $\eta_{c}$ of the dispersed and continuous phases, respectively. It was shown that in this case a pair of symmetrical counterrotating vortices emerges, whose rotation axes are opposite to those of the detached microdroplet moving freely in microchannel. A similar system was studied numerically in ${ }^{58}$. A pair of symmetric vortices emerging at early stage of microdroplet formation was revealed as well. Attention was paid to the fact that a flow pattern in a microdroplet in formation in the flow-focusing junction and co-flow microchannels may depend on wetting of capillaries by the dispersed phase ${ }^{59}$. The circulation was less intensive in the surfactant-laden flow-focusing device ${ }^{60}$. For the same system, the flow fields in the jetting mode at small and large capillary numbers were investigated as well ${ }^{61}$. The vortex flow inside a microdroplet during its formation in a capillary coflow was investigated in more details in ${ }^{62}$ by means of $\mu$ PIV method. It was found that vortex intensity in this case does not depend on the flow rate of the dispersed phase, but strongly depends upon interfacial tension, viscosity, and flow rate of the continuous phase. Flow patterns in a microdroplet during its formation in a T-shaped capillary was studied in ${ }^{63,64}$. For a significantly different dispersed and continuous phases flow rates $\left(q_{d}=0.001\right)$, a single vortex formation was observed, which intensity increases and reaches a maximum before droplet detachment. The mixing efficiency of droplet formation in different T-junction microchannels was also studied numerically ${ }^{65}$.

In spite of existing studies, many questions remain open. For co-flow microchannels, the peculiarities of the vortex intensity and size evolution during microdrop formation at different times require a detailed study. In particular, the correlation between the cross-section area of a neck generated during microdroplet formation ${ }^{36,66,67}$ and the vortex flow pattern in the droplet is still not a fully solved problem. The issue of the interrelation of vortex structures both during microdroplet formation and after its detachment requires attention as well. In the present work, the above-mentioned problems are considered for the first time in co-flow microchannels. The vortex flow in a microdroplet during its formation and after detachment is studied by means of $\mu$ PIV measurement for different flow rates of the dispersed and continuous phases. Experimental data are analyzed using the free software package PIVlab $^{68}$, implemented on the basis of MATLAB ${ }^{\circledR}$ as well as a simple theoretical model for pure swirling flow excluding shear flow contribution.

\section{EXPERIMENTAL}

\section{A. Microfluidic system and materials}

The schematic drawing of the microfluidic system used in this work is depicted in Fig. 1 where a PEEK T-junction was used to promote the co-flow. A silica capillary with inner and outer diameters $d_{d}^{i n}=150 \mu \mathrm{m}$ and $d_{d}^{\text {out }}=360 \mu \mathrm{m}$, respectively, was inserted into the left side of the T-junction and used for supplying the dispersed liquid. The continuous 


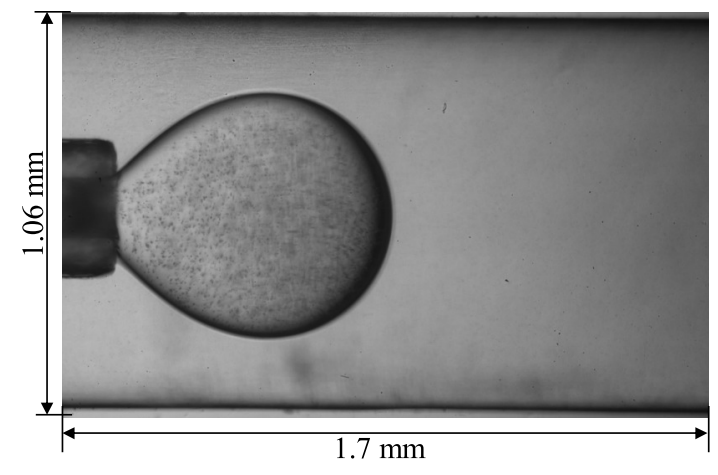

FIG. 2. Typical raw image of the droplet with seeded particles.

TABLE I. Continuous phase parameters.

\begin{tabular}{cccc}
\hline \hline $\begin{array}{c}\text { Continuous } \\
\text { phase number }\end{array}$ & $\begin{array}{c}\text { Glycerol volume } \\
\text { fraction, vol. } \%\end{array}$ & $\begin{array}{c}\text { Kinematic } \\
\text { viscosity, cSt }\end{array}$ & $\begin{array}{c}\text { Viscosity } \\
\text { ratio, } m\end{array}$ \\
\hline 1 & 80.27 & 94 & 0.053 \\
2 & 87.45 & 217 & 0.023 \\
3 & 92.7 & 445 & 0.011 \\
\hline \hline
\end{tabular}

medium was fed through the orthogonal port of the T-junction into a coaxial PTFE outer capillary with an inner diameter of $d_{c}^{i n}=1.06 \mathrm{~mm}$. Fluid flow rates were controlled by means of two syringe pumps (Cetoni, neMESYS 290N).

To minimize optical distortions due to the difference of index of refraction between the PTFE and air and also caused by the cylindrical shape of the external PTFE capillary, the latter was tightly inserted into a PDMS slab with a cylindrical channel of diameter equal to $1.06 \mathrm{~mm}$ (Fig. 1). The PDMS slab was produced using the SYLGARD ${ }^{T M} 184$ kit (Dow Corning), a material often used for microfluidics microsystems. Due to its high transparency and planar outer surface, such a slab made it possible to significantly reduce optical distortions. The length of the inner silica capillary was adjusted so that its tip is located inside the PDMS slab to generate a co-flow of the dispersed and continuous fluids (See Fig. 1).

Low-viscosity silicone oil with kinematic viscosity of $5 \mathrm{cSt}$ (Dow Corning, USA) has been used as the dispersed fluid. The continuous phases were mixtures of distilled water and glycerol. Their volume fractions and kinematic viscosities are presented in Table I along with the dispersed to continuous phases' viscosity ratios $\left(m=\eta_{d} / \eta_{c}\right)$. The interfacial tension $\sigma$ between silicon oil and water/glycerol mixture weekly depends on glycerol concentration ${ }^{69,70}$. Its mean value is about $30 \mathrm{mN} / \mathrm{m}$. The experiments were carried out at ambient temperature.

For $\mu$ PIV measurements, silicon oil was seeded with hydrophobic polyethylene microspheres of 1-4 $\mu \mathrm{m}$ (Cospheric, United States) at a concentration of 0.05 wt.\%. The microspheres were supplied as a dry powder. The polyethylene particles were dispersed homogeneously in silicone oil with an ultrasonic homogenizer (Bandelin, Sonopuls UW 2200).

\section{B. $\mu \mathrm{PIV}$ measurements}

We used a Nikon Eclipse TS100 inverted microscope with a 10x/0.25 objective and a Phantom Miro LAB 3a10 high-speed camera with frame rates between 160-1600 fps which corresponds to interframe time in the range of 0.625 to $6.25 \mathrm{~ms}$. The exposure time of the measurements was of $5 \mu \mathrm{s}$. The camera resolution was $1280 \times 800$ pixels, which allowed to cover the channel length of $1.7 \mathrm{~mm}$, which corresponded to the pixel physical resolution of about $1.3 \mu \mathrm{m}$. But since we used these seeding particles for back-light illumination, the fact that some particles are smaller this resolution did not impact the results of the cross-correlation used for the calculation of the vector field. Fig. 2 shows a typical raw image of the seeded droplet during its formation. It can be seen that the upper and lower parts of the inner and outer capillaries are in the focal plane of the microscope and that the distance between them is the same which indicates coaxiality of the capillaries. This setup allowed investigating flow patterns in the central section of the droplet. It is worth noting that the depth of field (focal plane thickness) of our optical set-up is very thin. In the case of volume illumination, it can be estimated as ${ }^{71} \delta_{z}=\frac{n \lambda_{0}}{N A^{2}}+\frac{n e}{N A \cdot M}$, where $n$ is the refraction index between the objective and the microfluidics device, $\lambda_{0}$ the mean wavelength of white light (on average $550 \mathrm{~nm}), N A$ the numerical aperture, i.e. 0.25 for our objective, $M$ the total magnification of the system $(\times 10$ for our objective), and $e$ the smallest distance that can be resolved by the camera on the image plane of the microscope (pixel resolution). The first term in this relationship corresponds to the depth of field resulting from diffraction effects, and the second term to contribution from geometrical effects. As a result, the depth of field is $14 \mu \mathrm{m}$, what is more than an order of magnitude lower than the droplet size. This ensures that by focusing in the mid-plane, off focus particles will have similar velocity than in-focus particles and will not perturbed the accuracy of the measurement. The obtained images were analyzed using the free software package PIVlab ${ }^{68}$ implemented in MATLAB ${ }^{\circledR}$ package.

Note that $\mu \mathrm{PIV}$ analysis of droplet formation is a rather complex and time-consuming procedure. This is attributed both to high velocity gradients in a droplet and to spatial variations in its size and shape. During droplet formation it is necessary to control the time step as well as the investigated area not only for each experiment, but also for each time moments within the same experiment since the instantaneous flow field needs to be measured.

Before data-processing procedure, the Contrast Limited Adaptive Histogram Equalization filter (CLAHE) ${ }^{72}$ has been used for increasing the readability of the image data. For the cross-correlation part of analysis, the iterative Fast Fourier Transport (FFT) approach was applied ${ }^{15,73}$. To do that, the mean displacement of the seeding particles has been calculated first based on an Interrogation Area (IA) of 64x64 pixels. Then, the initial IA was divided into smaller 32x32 IA's, which initial value was taken as the displacement calculated in the previous step and then calculated again for these smaller IA and deformed according to the local velocity gradients in 


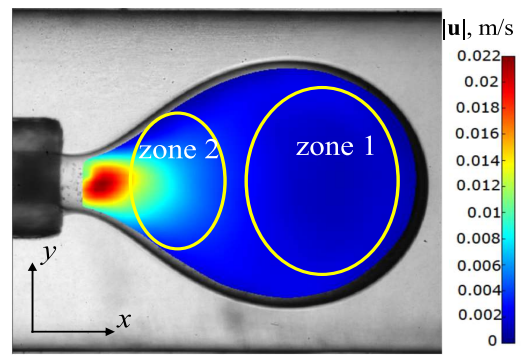

(a)

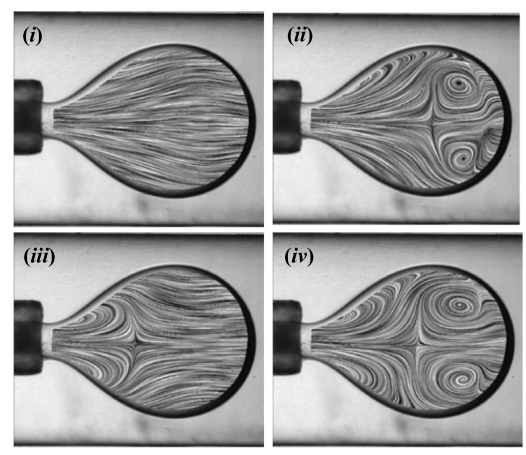

(b)

FIG. 3. Flow patterns in the silicon droplet's central longitudinal section: (a) modulus of the velocity vector field, (b) LIC visualization of streamlines for (i) the original velocity field $\mathbf{u}$, (ii) velocity perturbations $\mathbf{u}_{1}^{\prime}$, (iii) velocity perturbations $\mathbf{u}_{2}^{\prime}$, and (iv) high-passed velocity perturbations $\mathbf{u}^{\prime}$.

the image. The intensity peak of the correlation matrix inside each interrogation area was determined with Gaussian $2 \times 3$ point fit function. Note that in the velocity range between 1 to $20 \mathrm{~mm} / \mathrm{s}$ used in our experiments the relative uncertainty of displacement measurements ${ }^{15,74,75}$ of the particle image ensembles within IA was ranged from 2 to $6 \%$.

\section{Flow field visualization}

Analysis of raw images with seeded particles allows deriving two-dimensional velocity vector field $\mathbf{u}=\left(u_{x}, u_{y}\right)$ in the central longitudinal section of a droplet which is in the focal plane of the inverted microscope. Fig. 3a shows the field of velocity values in the droplet, coded with a color scale in the laboratory frame of reference. It can be seen that the velocity field is significantly inhomogeneous. To see flow patterns, the LIC (Line Integral Convolution) visualization technique has been applied (Fig. 3b), allowing to analyze the flow structure in the droplet symmetry plane, cutting it into two halves along its length ${ }^{76}$. Fig. $3 b(i)$ presents a flow pattern plotted on the basis of velocity vectors as calculated from $\mu$ PIV measurements. This image, however, does not allow to see vortex formations. This is due to the fact that the measured velocity field includes the droplet translational velocity which is attributed to increasing droplet size and neck stretching. Subtracting the average droplet velocity from the measured velocity field allows one to visualize the vortex flow in a droplet. The heterogeneity of the velocity field shown in Fig. 3a hinders identification of flow structure within different areas of the microdroplet. In the given case, a region with reduced velocity (zone 1) is formed around the droplet center, while a zone with increased velocity (zone 2 ) is created close to the neck. In particular, at flow rates $Q_{c}=0.1 \mathrm{~mL} / \mathrm{min}$ and $Q_{d}=0.01 \mathrm{~mL} / \mathrm{min}$ of continuous and dispersed phases, the mean velocity vector in zone 1 is equal to $\bar{u}_{1}=0.0015 \mathrm{~m} / \mathrm{s}$ while in the zone 2 it corresponds to $\bar{u}_{2}=0.004 \mathrm{~m} / \mathrm{s}$. Fig. $3 \mathrm{~b}$ (ii) shows the streamlines pattern which corresponds to velocity perturbations $\mathbf{u}_{1}^{\prime}=\mathbf{u}-\overline{\mathbf{u}}_{1}$. It can be seen that subtracting the mean velocity $\overline{\mathbf{u}}_{1}$ allows us to identify the vortex flow in the zone 1. On the other hand, the flow pattern corresponding to the velocity perturbation defined as $\mathbf{u}_{2}^{\prime}=\mathbf{u}-\overline{\mathbf{u}}_{2}$, is presented in Fig. 3b(iii). It reveals an eddy flow near the neck (zone 2), which is almost invisible in Fig. 3b(ii).

Thus, in the case of a highly inhomogeneous velocity distribution in a droplet, subtracting a certain constant velocity inevitably leads to the loss of some information about the vortex flow in it (cf. Fig. 3b(ii) and 3b(iii)). To solve this problem, we used a high-pass filter for the original vector velocity field: the mean velocity over some rectangular subdomain is subtracted from each part of the total velocity field ${ }^{77}$. In other words, the low frequency flow patterns should be removed highlighting the local velocity patterns as vortices. The high-passed flow pattern is represented in Fig. $3 \mathrm{~b}(\mathrm{iv})$. As can be seen, the vortex structures of local velocity disturbances are clearly visible in both zones.

\section{EVALUATION OF VORTEX INTENSITY}

Visualization of the vortex flow patterns inside the droplet, however, does not reveal the vortex intensity. Such a quantification can be made from data obtained from $\mu$ PIV analysis, allowing to calculate both the two-dimensional velocity field $\mathbf{u}=\left(u_{x}, u_{y}\right)$ of the dispersed fluid and the velocity gradient tensor $\nabla \mathbf{u}=\left(\partial_{x} u_{x}, \partial_{y} u_{x} ; \partial_{x} u_{y}, \partial_{y} u_{y}\right)$ where the definition $\partial_{i} u_{k} \equiv \frac{\partial u_{k}}{\partial x_{i}}$ was used here. This flow field is measured in the plane $(x, y)$ of symmetry of the droplet which contains the axis of symmetry of the droplet as well as the axis of symmetry of both capillaries (see Fig 3a). This implies that the velocities orthogonal to the plane of observation are expected to be zero due to this axial symmetry of the system. The velocity gradient tensor $\nabla \mathbf{u}$ contains complete information about deformation and rotation behavior of fluid elements inside a droplet. The traditional way to separate strain and rotation is to decompose the velocity gradient tensor into symmetric and antisymmetric parts as $D_{i k}=\frac{1}{2}\left(\partial_{i} u_{k}+\partial_{k} u_{i}\right)$ and $W_{i k}=\frac{1}{2}\left(\partial_{i} u_{k}-\partial_{k} u_{i}\right)$, respectively. The symmetric $\mathbf{D}$ tensor corresponds to the strain rate tensor while the antisymmetric $\mathbf{W}$ one is called the vorticity tensor.

The dual vector of the vorticity tensor $\omega=$ $-\left(W_{y z} \mathbf{i}+W_{x z} \mathbf{j}+W_{x y} \mathbf{k}\right)$ is known as the angular velocity oriented in the principal direction of strain rate tensor $\mathbf{D}$. In particular, the simple shear flow $u_{x}(y)$ in $(x, y)$ plane includes 
(a)

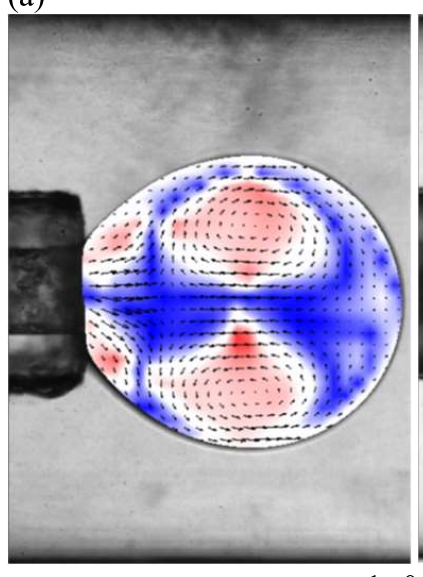

(b)

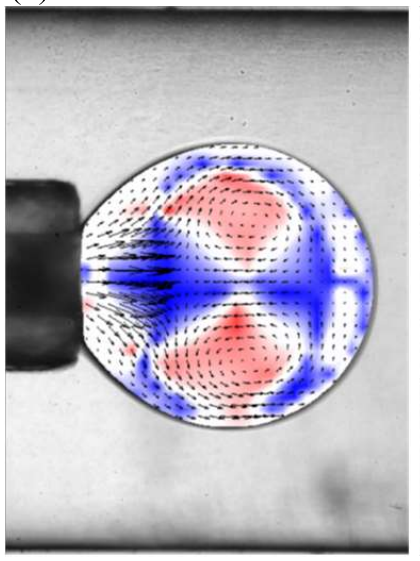

(c)

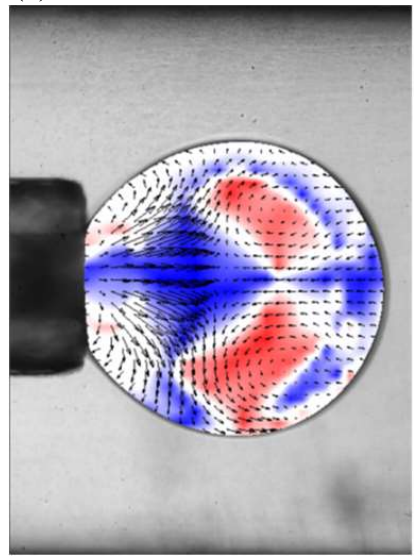

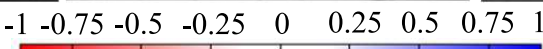

FIG. 4. Field of flow-type parameter $\Psi$ for $Q_{c}=0.1 \mathrm{~mL} / \mathrm{min}$ and $Q_{d}=0.005$ (a), 0.01 (b) and 0.025 (c) $\mathrm{mL} / \mathrm{min}$. The dispersed continuous phase viscosity ratio is $m=0.023$.
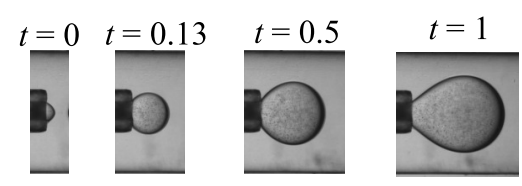

(a)
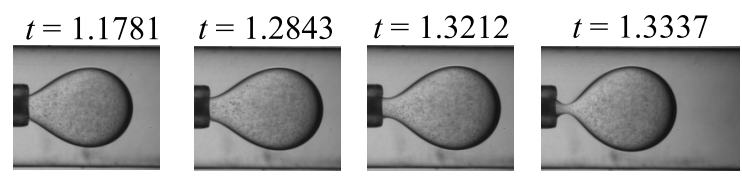

(b)
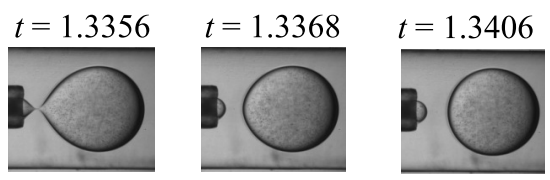

(c)

FIG. 5. Different stages of the formation of a silicone oil droplet coflowing with water-glycerol mixture in coaxial capillaries at $Q_{c}=$ $0.1 \mathrm{~mL} / \mathrm{min}, Q_{d}=0.01 \mathrm{~mL} / \mathrm{min}$, and $m=0.023$ : (a) droplet growth, (b) neck formation, (c) droplet detachment. Instants time $t$ are expressed in seconds.

part of rotation of a liquid element with the angular velocity $\omega=-\frac{1}{2} \partial_{y} u_{x}=-\frac{1}{2} \dot{\gamma}$. So, the curl of a velocity is not suitable for estimating the vortex flow intensity because it does not distinguish the pure rotational element of the fluid from a simple shear flow. To solve this problem, various vortex evaluation techniques were proposed ${ }^{78-83}$. The most common ways of estimating the net rotation intensity of a fluid element are $Q$ and $\lambda_{c i}$ criteria $^{81,82}$. The first consists of determining the flow domain of an incompressible fluid with a positive value of the second invariant $Q=\frac{1}{2}\left[\operatorname{tr}(\nabla \mathbf{u})^{2}-\operatorname{tr}\left(\nabla \mathbf{u}^{2}\right)\right]$ of the velocity gradient tensor $\nabla \mathbf{u}$. In this area, streamlines form the closed trajectories corresponding to the vortex flow ${ }^{84}$. The greater the value of $Q$, the larger is the rotational intensity of a fluid. The second criterion requires solving the characteristic equation $\lambda^{3}+P \lambda^{2}+Q \lambda+R=0$ for the eigenvalues of $\nabla \mathbf{u}$ tensor, where $P=-\operatorname{tr}(\nabla \mathbf{u})$ and $R=-\operatorname{det}(\nabla \mathbf{u})$ correspond to the first and third invariants of the tensor. For an incompressible fluid, the first invariant $P$ is zero. According to this criterion, pure rotation takes place when the eigenvalues are complex-conjugate $\lambda_{1,2}=\lambda_{r} \pm i \lambda_{c i}$. In this case the period of a fluid particle orbit is defined by the imaginary part as $2 \pi / \lambda_{c i}{ }^{84}$.

However, in a recent work ${ }^{85}$ it was proved that the $Q$ and $\lambda_{c i}$ criteria are strongly contaminated by shearing. In addition, in the general case they do not contain information about the vorticity direction. As a result, a new technique was developed to estimate the vorticity intensity at a given point, which is based on drawing the so-called rortex vector ${ }^{85-87}$. The rortex vector is a part of the vorticity vector representing the rigidly rotating vector. This vector is not influenced by shear flow while its direction matches the vorticity direction and its value is equal to the doubled angular rotation velocity of the selected fluid element. The procedure of finding the rortex vector consists of two parts: (i) searching the rotation axis and (ii) determining the angular velocity of the fluid element in the plane perpendicular to this vector. Since this paper considers the two-dimensional flow in the central section of a droplet, the rotation axis is always perpendicular to the flow plane, so the problem is limited to calculating the value of the rortex vector, which corresponds to the angular velocity sought. This method is based on the following statement: the pure rotation without shear flow modes (swirling flow) of a fluid element under consideration takes place if and only if the inequality $g_{z}=-\left.\left.\partial_{x} u_{y}\right|_{\theta} \partial_{y} u_{x}\right|_{\theta}>0$ takes place at any rotation angles $\theta$ of the reference system. The above inequality is satisfied if 
$\beta^{2}-\alpha^{2}>0^{87}$, where

$$
\begin{aligned}
& \alpha=\frac{1}{2} \sqrt{\left(\frac{\partial u_{y}}{\partial y}-\frac{\partial u_{x}}{\partial x}\right)^{2}+\left(\frac{\partial u_{y}}{\partial x}+\frac{\partial u_{x}}{\partial y}\right)^{2}} \\
& \beta=\frac{1}{2}\left(\frac{\partial u_{y}}{\partial x}-\frac{\partial u_{x}}{\partial y}\right)
\end{aligned}
$$

in the laboratory frame of reference. As the angular velocity of a fluid element, the minimum value of $\omega=\left.\frac{\partial u_{x}}{\partial y}\right|_{\theta}$ is considered $^{87}$ :

$$
\omega_{\text {rort }}=\omega_{\theta, \min }= \begin{cases}\beta-\alpha, & \text { if } \beta>0 \text { and } \beta^{2}-\alpha^{2}>0 ; \\ \alpha-\beta, & \text { if } \beta<0 \text { and } \beta^{2}-\alpha^{2}>0 ; \\ 0, & \text { if } \beta^{2}-\alpha^{2} \leq 0 .\end{cases}
$$

Using such an approach makes it possible to isolate areas of pure swirling flow as well as to estimate the angular rotation velocity of any fluid element.

Let's consider the relative contribution of shear and rotational flow inside the droplet during formation. For this purpose the flow-type parameter $\Psi=(|\mathbf{D}|-|\mathbf{W}|) /(|\mathbf{D}|+|\mathbf{W}|)$ was introduced where $|\mathbf{D}|=\left(D_{i k} D_{i k}\right)^{1 / 2}$ and $|\mathbf{W}|=$ $\left(W_{i k} W_{i k}\right)^{1 / 2}$ are magnitudes of the strain rate and vorticity tensors, respectively (the repeated indices are implicitly summed). In the case of pure rotational flow $\Psi=-1, \Psi=0$ for pure shear flow, and $\Psi=1$ for pure plane extensional flow. Intermediate values indicate a mixed type of flow. The field of the flow-type parameter $\Psi$ is presented in Fig. 4 for the fixed flow rate of continuous phase $Q_{c}=0.1 \mathrm{~mL} / \mathrm{min}$ and different flow rates of the dispersed phase $Q_{d}=0.005$ (a), 0.01 (b) and 0.025 (c) $\mathrm{mL} / \mathrm{min}$ during the droplet formation. The time moment for each $Q_{d}$ value is chosen so that volumes of droplets are consistent. In this figure, the corresponding velocity vector field is displayed as well. It follows that in the vortex flow regions the values of parameter $\Psi$ are ranged between -1 and 0 , which indicates the mixed type of flow including both rotational and shear components. It is seen that as the flow rate of the dispersed phase $Q_{d}$ increases, the contribution of shear flow in the vortex zones decreases. The evidence of the mixed type of flow in the vortex zones may be a reason for an incorrect estimation of intensity of pure vortex flow. Taking this into account, we used another method based on the estimation of the rortex vector values allowing to exclude the shear flow influence.

\section{RESULTS AND DISCUSSION}

\section{A. Stages of droplet formation}

Fig. 5 shows evolution of shape and size of a growing droplet at flow rates $Q_{c}=0.1 \mathrm{~mL} / \mathrm{min}$ and $Q_{d}=0.01 \mathrm{~mL} / \mathrm{min}$, and viscosity ratio $m=0.023$. This process can be subdivided into the following three stages: (i) droplet growth (Fig. 5a), (ii) neck formation (Fig. 5b), and (iii) neck rupture and droplet detachment (Fig. 5c).

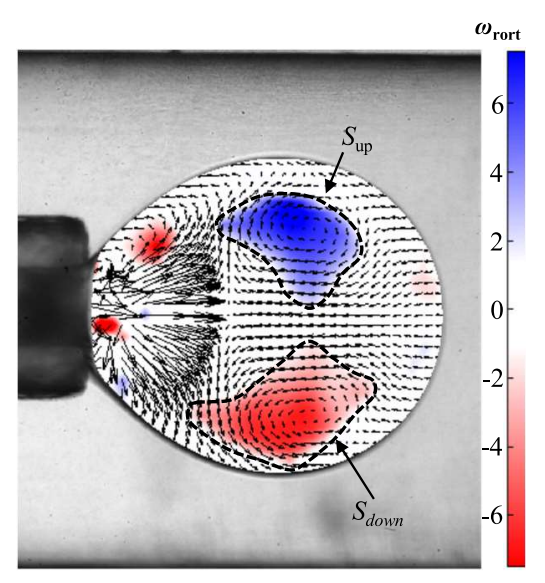

(a)

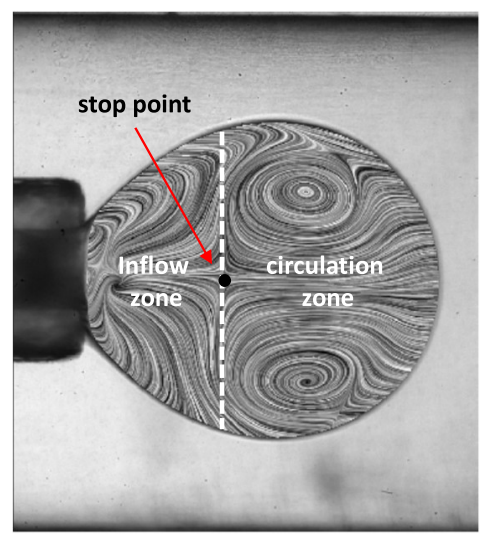

(b)

FIG. 6. Flow patterns in a growing droplet of $m=0.023$ for $Q_{c}=$ $0.1 \mathrm{~mL} / \mathrm{min}$ and $Q_{d}=0.025 \mathrm{~mL} / \mathrm{min}$ : (a) velocity vector and angular velocity $\omega_{\text {rort }}$ fields as vortex zones $S_{u p}$ (blue) and $S_{\text {down }}$ (red), (b) LIC representation of streamlines. The color scale indicates magnitudes and sign of angular velocity $\omega_{\text {rort }}$.

The first stage is the longest part of droplet formation process (Fig. 5a), where the motion of the droplet center of mass is driven mainly by its growth. The second stage is related to the neck formation. This stage is much faster. In this stage the droplet volume does not virtually change. During this stage the droplet starts moving away from the inner capillary under the action of the flowing continuous phase while the neck is stretching and thinning (Fig. 5b). The third stage includes the rupture of the neck and the droplet detachment and its motion along the outer capillary (Fig. 5c). It can be seen that right after the detachment the droplet takes a pointed shape at the neck junction, which then quickly transforms into a steady shape. 


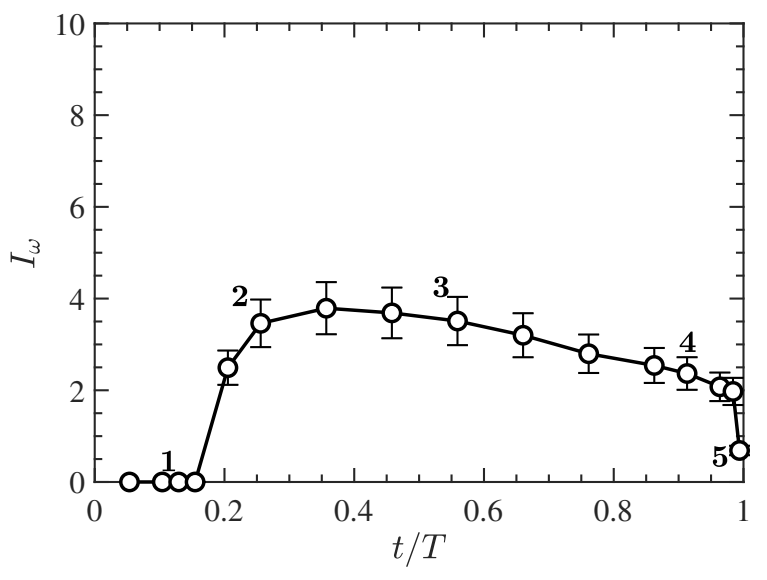

(a)

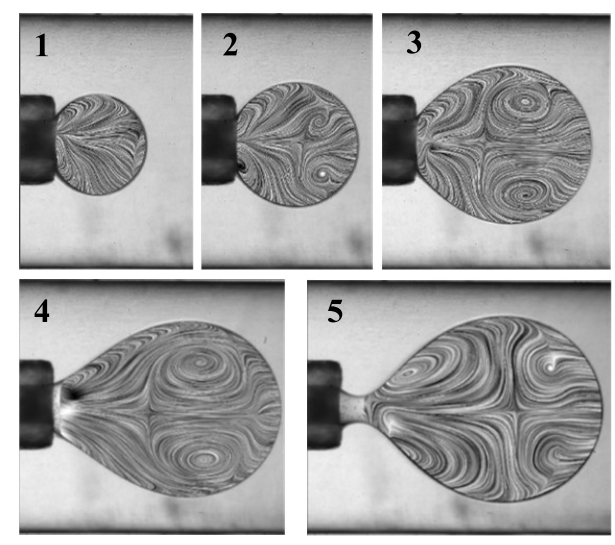

(b)

FIG. 7. Evolution of the vortex flow intensity $I_{\omega}$ (a) and vortex flow patterns (b) during drop formation at $Q_{c}=0.1 \mathrm{~mL} / \mathrm{min}$, $Q_{d}=0.025 \mathrm{~mL} / \mathrm{min}$, and $m=0.023$. The numbers of the flow patterns correspond to the numbers of the circle symbols.

\section{B. Vortex flow patterns during droplet formation}

\section{Droplet growth stage}

Fig. 6 shows typical flow patterns within a growing droplet and presents the velocity vector field (Fig. 6a) and streamlines in the LIC presentation (Fig. 6b). Symmetrical counterrotating vortex rings are observed in the upper and lower parts of the central longitudinal section of the droplet. The vortex rotates clockwise in its upper part and counterclockwise in the lower part. It is reasonable to assume that due to the capillary axial symmetry, a similar flow vortex structure takes place in an arbitrary central longitudinal cross-section suggesting the formation a toroidal vortex.

Figs. $6 \mathrm{a}$ and $6 \mathrm{~b}$ illustrate the angular velocity field $\omega_{\text {rort }}$ of pure swirling flow calculated according to Eqs. (1) and (2). The white field in Fig. 6a corresponds to the entire lack of swirling flow, while the blue and red colors indicate clockwise and counterclockwise rotation, respectively. These areas are consistent with the velocity vector field and the shape of the streamlines, indicating fluid motion along closed trajectories (Fig. 6b). It is seen that values of the angular velocity $\omega_{\text {rort }}$ are distributed inhomogeneously in these areas. For this reason, the average angular velocities in both of areas $S_{u p}$ and $S_{d o w n}$ were estimated:

$$
\begin{aligned}
& \bar{\omega}_{u p}=\frac{1}{S_{u p}} \int_{S_{u p}}\left|\omega_{\text {rort }}\right| d S, \\
& \bar{\omega}_{\text {down }}=\frac{1}{S_{\text {down }}} \int_{S_{\text {down }}}\left|\omega_{\text {rort }}\right| d S .
\end{aligned}
$$

Obviously, due to the axial symmetry of the system, the obtained mean angular velocities should be nearly equal, while the difference between them is due to measurement errors. To smooth out such errors at any time moment, we introduced the following mean parameter

$$
I_{\omega}=\frac{1}{2}\left(\bar{\omega}_{u p}+\bar{\omega}_{d o w n}\right),
$$

which will be named 'the intensity of the vortex flow'. The LIC representation of the streamlines (Fig. 6b) shows clearly that during droplet formation, the corresponding flow field in it can be subdivided conditionally into the inflow and circulation domains. The boundary between them is represented by the line including a stop point where elongational flow is important (see the dotted line in Fig. 6b).

Dependence of vortex flow intensity $I_{\omega}$ in a droplet on the dimensionless time $\widetilde{t}=t / T$ is displayed in Fig. 7 ( $T$ is the time at droplet detachment). In this case flow rates of continuous and dispersed phases were taken as $Q_{c}=0.1 \mathrm{~mL} / \mathrm{min}$ and $Q_{d}=0.025 \mathrm{~mL} / \mathrm{min}$, and the viscosity ratio between the dispersed and continuous phase is $m=0.023$. Numbers denote time moments corresponding to LIC representations of flow patterns given below. It follows from here that in the beginning a vortex-free flow is generated in the growing droplet (see flow pattern 1 in Fig. 7). As the droplet size increases, a circulation zone occurs where vortex flow intensity $I_{\omega}$ sharply increases, reaches a maximum value, and then slowly decreases. Just before the droplet detachment, the vortex intensity in the circulation zone drops down, while a new pair of symmetric vortices is formed in the vicinity of the neck (see flow pattern 5). These vortices will be considered in more details in sections IV B 2 and IV C.

It is worth noting that at different flow rates $Q_{d}$ of the dispersed phase, the size of the circulation zone for the same volume $\left(Q_{d 1} t_{1}=Q_{d 2} t_{2}=Q_{d 3} t_{3}\right)$ of growing droplets is variable. It is observed in Fig. 8 at the given flow rate $Q_{c}=0.1 \mathrm{~mL} / \mathrm{min}$ of the continuous phase for three relative flow rates of the dispersed phase $q_{d}=0.01,0.05$ and 0.25 . It is seen that increasing $Q_{d}$ leads to decreasing area of the circulation zone and, on the contrary, increasing inflow zone area. Therefore, the observed growth of the inflow zone allows one to conclude that the flow structure to the left of the stop point is caused mainly by feeding the drop with dispersed liquid coming from the inner capillary. 
(a)

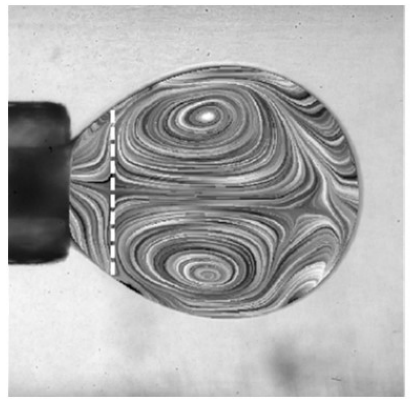

(b)

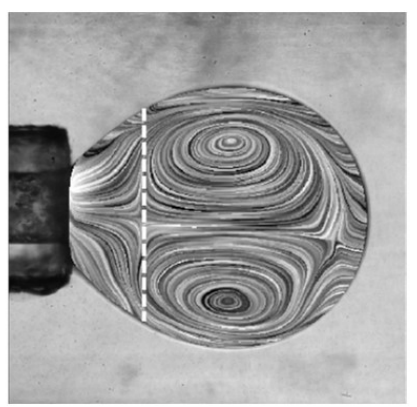

(c)

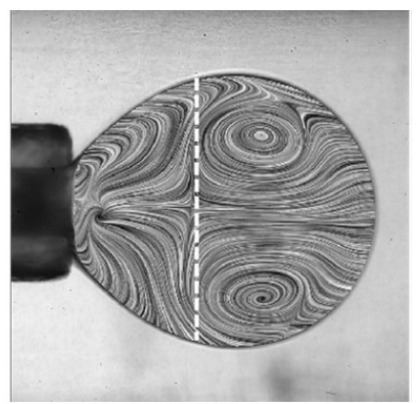

FIG. 8. LIC representation of flow patterns in droplets formed at a given continuous phase flow rate $Q_{c}=0.1 \mathrm{~mL} / \mathrm{min}$ and different relative flow rates of the dispersed phase $q_{d}=0.01$ (a), 0.05 (b) and 0.25 (c). The viscosity ratio between the dispersed and continuous phase is $m=0.023$.

For a more quantitative investigation of vortices present during droplet formation, the velocity of the droplet's front point as well as flow velocities along the vortex streamlines were estimated. To do so, we assumed that a droplet takes a spherical shape at each time. The fluid velocity field in a droplet is formed due to its feeding by the dispersed fluid as well as by the motion of the continuous phase which is applying shear stress on the droplet interface. An increase of droplet volume affects the radial velocity components, whereas the flow of continuous phase induces motion of the dispersed fluid in the tangential direction. Taking into account that the volume of a growing droplet is equal to $V_{d}(t)=Q_{d} t$, its radius can be estimated as

$$
r_{d}(t)=\left(k Q_{d} t\right)^{1 / 3},
$$

where $k=3 / 4 \pi$ for a spherical droplet. Fig. 9 shows the measured time dependencies of the droplet radius for four dispersed phase flow rates $Q_{d}=0.005,0.01,0.025,0.05 \mathrm{~mL} / \mathrm{min}$ and two continuous phase flow rates $Q_{c}=0.1$ (Fig. 9a) and $0.2 \mathrm{~mL} / \mathrm{min}$ (Fig. 9b). It can be seen that increasing the dispersed phase flow rate leads to a noticeable decrease of the droplet formation time $T$ (Fig. 9c, solid curves) and an insignificant increase of its size $r_{d}(T)$ at the detachment moment from the inner capillary (Fig. 9c, dashed curves). The results obtained are in agreement with the numerical simulations and experimental data from the literature ${ }^{36,63}$. The introduction of the dimensionless length $\widetilde{r}_{d}=r_{d} / r_{d}(T)$ transforms Eq. (5) to the following dimensionless form

$$
\widetilde{r}_{d}(\widetilde{t})=\widetilde{t}^{1 / 3}
$$

which is independent of the continuous and dispersed fluid flow rates. Converting the experimental data in Figs. 9a and $9 \mathrm{~b}$ to a dimensionless form results in a master curve for any continuous and dispersed phase flow rates $Q_{c}$ and $Q_{d}$ (see Fig. 9d). The master curve agrees well with the red dashed curve obtained from Eq. (6). The experimental data are slightly below the theoretical predictions after the time $\tilde{t}>0.6$. This is caused by the assumption of spherical droplet shape. The difference is seen in the inset of Fig. 9d showing that a small portion of the dispersed fluid (highlighted in red) is outside the sphere most adjacent to the droplet interface.

The experimental time dependence of the ratio $\widetilde{U}_{f p}=$ $U_{f p} / U_{c}$ of the droplet's front point (red dot in the inset) velocity $U_{f p}$ to the average velocity $U_{c}=\frac{4 Q_{c}}{\pi d_{c}^{2}}$ of the continuous phase is presented in Fig. 10. It is seen that at the beginning of the droplet formation the dimensionless velocity $\widetilde{U}_{f p}$ is quite high but then quickly decreases, and before the detachment (at the moment $\widetilde{t} \cong 0.9$ ) this velocity increases again.

Before the neck formation, the motion of the droplet interface is mainly associated with the growth of its volume (see Figs. 5 and 7). For this time interval, the interface velocity can be estimated from Eq. (5): $U_{r}(t)=d r_{d}(t) / d t=$ $\frac{1}{3}\left(k Q_{d}\right)^{1 / 3} t^{-2 / 3}$. Taking into account that the droplet leftmost point is pinned to the inner capillary, the velocity of droplet center of gravity along the capillary axis coincides with the droplet swelling velocity $U_{r}$, while velocity of the front point is equal to $U_{f p}=2 U_{r}$. The theoretical dependence of the relative velocity $U_{f p} / U_{c}$, on the dimensionless time corresponds to the dashed line in Fig. 10 and quantitatively agrees with the obtained data. This indicates that in a sufficiently wide time range, the frontal point velocity of the droplet is controlled by droplet swelling due to the feeding of dispersed fluid into the droplet. An increase of the frontal point velocity $U_{f p}$ at $t>0.9 T$ is related to the neck formation and beginning of the droplet motion under the action of the continuous phase which applies a shear stress on the droplet interface due to differences in mean velocities between the droplet and the continuous phase. This process correlates with reduction in the vortex flow intensity as shown in Fig. 7.

To study the flow velocity of the dispersed fluid in the vortex zones, variations of the tangential velocity $U_{v}^{\max }$ were examined in the upper (solid line) and lower (dashed line) parts of the droplet (Fig. 11b) along the closed streamlines as a function of the polar angle $\Theta$ (Fig. 11a). The polar angles $\Theta$ were counted from points number 1 (see Fig. 11b) in the range from 0 to $2 \pi$ along the highlighted arrows. As can be seen, the vortex flow velocity reaches its maximums at points 1 and 3 corresponding to $\Theta=0$ and $\pi$ while minima are observed at $\Theta=\pi / 2$ and $3 \pi / 2$ (points 2 and 4 ). Since the fluid flow pat- 


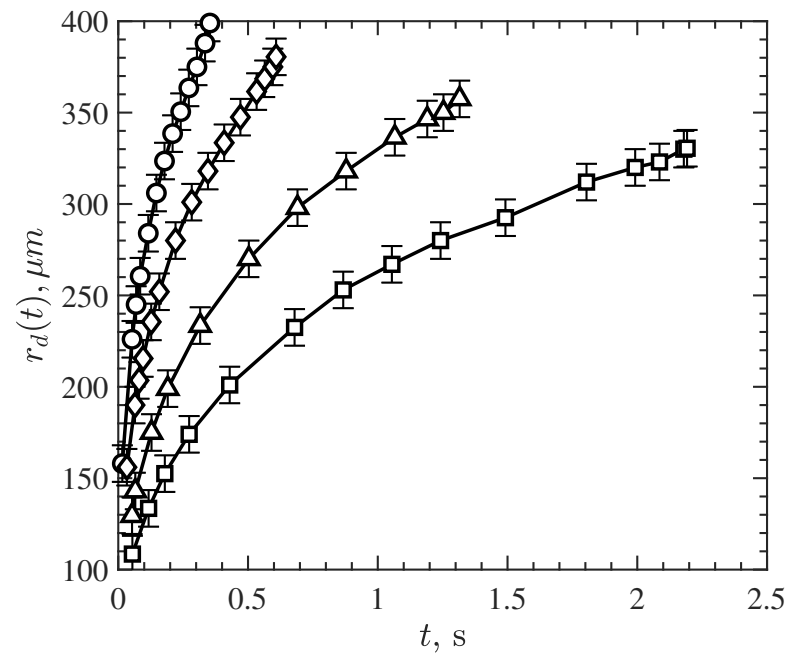

(a)

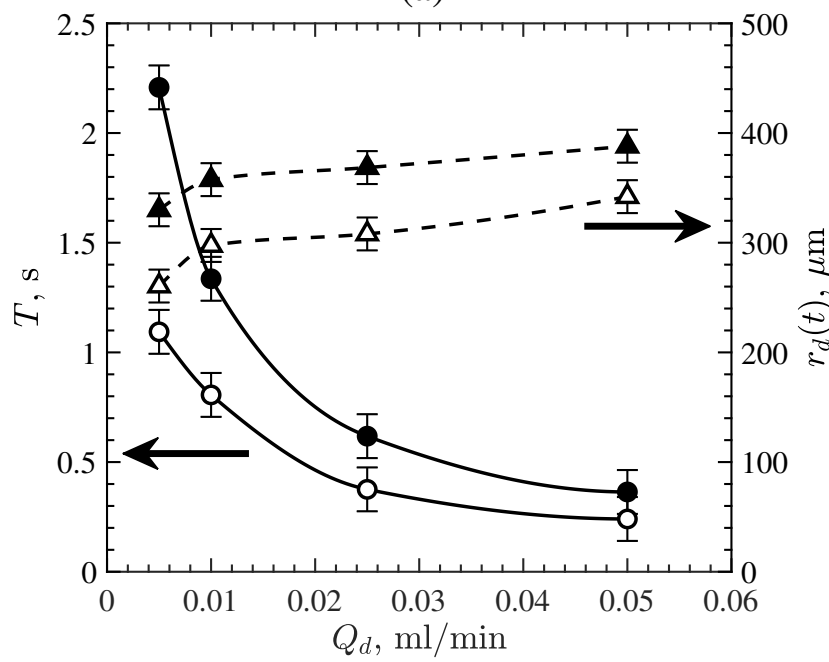

(c)

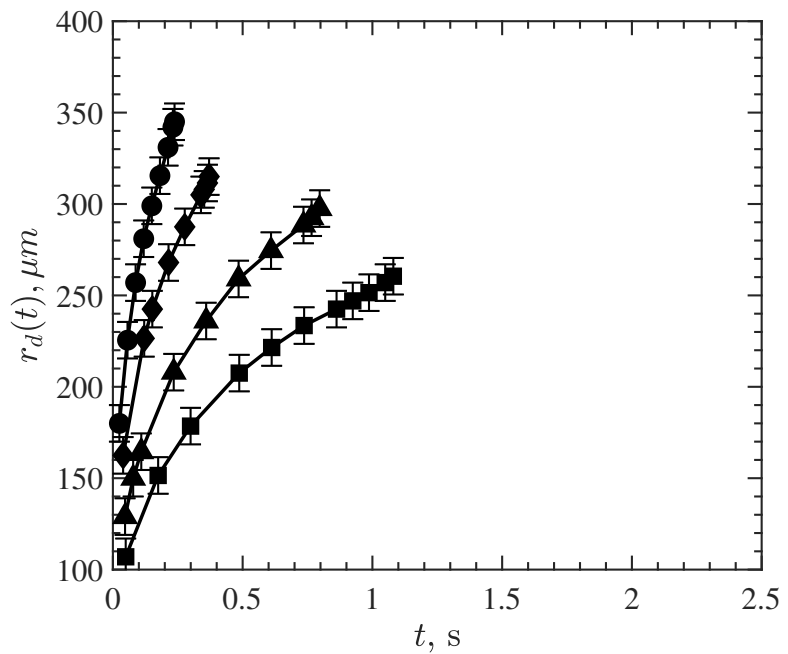

(b)

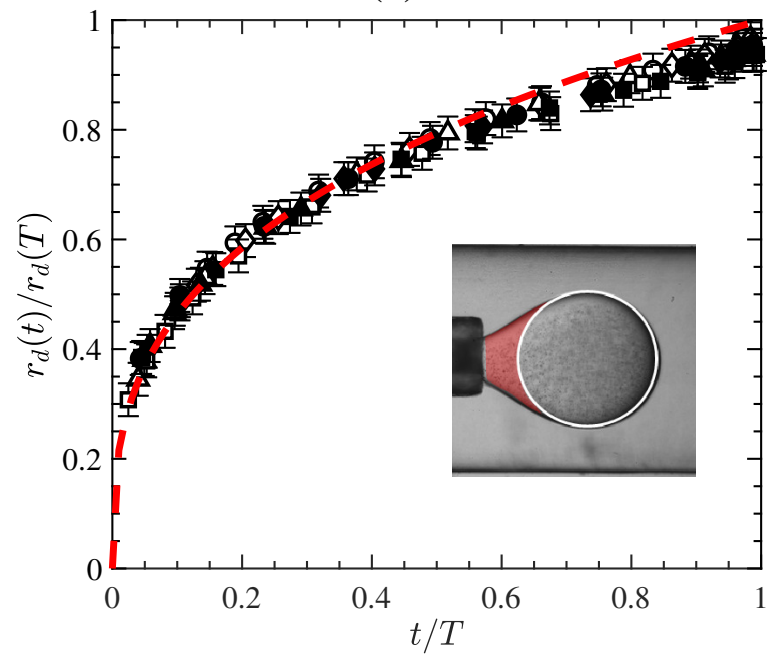

(d)

FIG. 9. Time dependence of measured droplet size for (a) $Q_{c}=0.1 \mathrm{~mL} / \mathrm{min}$ (open symbols), and (b) $Q_{c}=0.2 \mathrm{~mL} / \mathrm{min}$ (filled symbols) for various dispersed flow rates $Q_{d}=0.005$ (squares), 0.01 (triangles), 0.025 (diamonds) and $0.05 \mathrm{~mL} / \mathrm{min}$ (circles); dependencies of droplet formation time $T$ (left axis, solid lines, circles) and radius $r_{d}(T)$ (right axis, dashed lines, triangles) on flow rate $Q_{d}$ of the dispersed phase (c); dependence of dimensionless droplet size $r_{d} / r_{d}(T)$ on dimensionless time $t / T$ (d). The red dashed line corresponds to Eq. (6). The viscosity ratio between the dispersed and continuous phase is $m=0.023$.

tern in a droplet is axisymmetric, the differences between the curves presented comes from measurement uncertainties.

Such inhomogeneous behavior of the dispersed fluid velocity along the closed streamlines is associated with variations of the continuous fluid velocity along the droplet interface. Indeed, the increase in flow velocity in the vicinity of point 1 is attributed to its proximity to the wall of the outer capillary, where the velocity of the continuous phase is much higher than the average value. As the angle $\Theta$ increases, the local velocity of the continuous phase at the interface decreases due to its deviation from the main flow direction leading to a noticeable decrease in the fluid velocity in the neighborhood of points 2 and 4 . The maximum of local velocity at point 3 can be explained as follows. The dispersed fluid, which is not involved in the vortex flow near the interface is directed to- wards the front point and then rushes into the narrowing area between the two opposite vortices resulting in a local velocity increase around points 3 (yellow arrows in Fig. 11b ).

The maximum velocity of the continuous phase flow near point 1 can be estimated as

$$
U_{c}^{\max }(t) \approx \frac{Q_{c}}{\pi\left[r_{c}^{2}-r_{d}^{2}(t)\right]},
$$

where $r_{c}=0.53 \mathrm{~mm}$ is the outer capillary radius (Section II A), and the drop radius $r_{d}(t)$ is defined by Eq. (5). The condition of velocity continuity at the interfacial boundary along the direction of vortex rotation (Fig. 6a) indicates that the circulating fluid flow in the drop is formed mainly under the action of the continuous phase flow. The time de- 


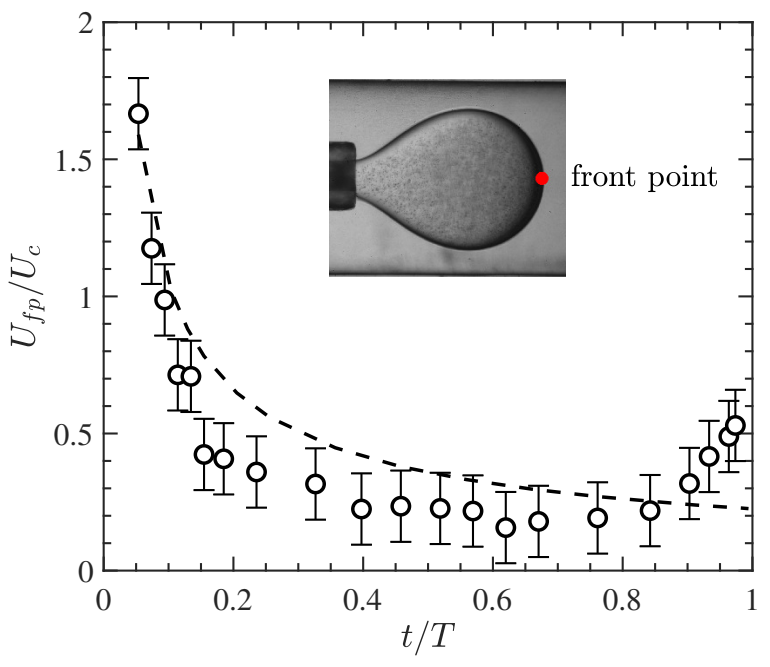

FIG. 10. Dependence of the relative velocity of the droplet front point $U_{f p} / U_{c}$ with time for $Q_{c}=0.1 \mathrm{~mL} / \mathrm{min}, Q_{d}=0.025 \mathrm{~mL} / \mathrm{min}$, and $m=0.023$. The circle symbols correspond to the measured data and dashed line stands for the theoretical estimate.

pendence of $U_{c}^{\max }(t)$ estimated from Eq. (7) is in qualitative agreement with the experimental measurements of the maximum tangential velocity of vortex motion $U_{v 1}^{\max }(t)$ measured at point 1 in the process of growing droplets for $Q_{c}=0.1$ and $0.2 \mathrm{~mL} / \mathrm{min}$ and different values of $Q_{d}$ (see Fig. 12a and 12b, respectively): the slopes of the theoretical and approximated curves are almost identical.

The fact that the velocity $U_{c}^{\max }(t)$ is above the velocity $U_{v 1}^{\max }(t)$ is explained by the existence of a pure shear flow layer between vortices and droplet interface (Fig. 4). Such a layer leads to a reduction of tangential velocity in the vorticity area. Therefore, the flow rate $Q_{d}$ of the dispersed phase is basically responsible for the radial velocity of the droplet interface which is related to a volume increase, while the flow rate $Q_{c}$ of the continuous phase influences the velocity of the vortex flow. It becomes clear from here that the vortex flow in a droplet should occur when the tangential velocity, influenced by the continuous phase velocity, dominates over the radial components. Introducing parameter $\Omega=U_{r} / U_{c}^{\max }$, it can be concluded that the dominating role in flow pattern formation in a droplet at $\Omega \ll 1$ is played by the continuous phase, whereas motion of the dispersed fluid at $\Omega \sim 1$ is determined by the droplet volume growth.

Fig. 13 presents time dependences of intensity $I_{\omega}(t)$ of pure vortex flow (left axis), and $\Omega(t)$ parameter calculated from the $\mu \mathrm{PIV}$ (right axis), flow field measurements for $Q_{c}=$ $0.1 \mathrm{~mL} / \mathrm{min}$ and different relative flow rates of the dispersed phase $q_{d}=Q_{d} / Q_{c}$, and a dispersed and continuous phases viscosity ratio $m=0.053$. It can be concluded that the vortex-free flow occurs for $\Omega<0.5$, whereas for $\Omega>0.5$ vortex-free flow takes place in the droplet. It can be seen that the vortex-free flow is formed during a certain initial time interval, which becomes longer while increasing the relative flow rate $q_{d}$ of the dispersed phase (see Fig. 13).

Fig. 13 shows that the average vortex angular velocity

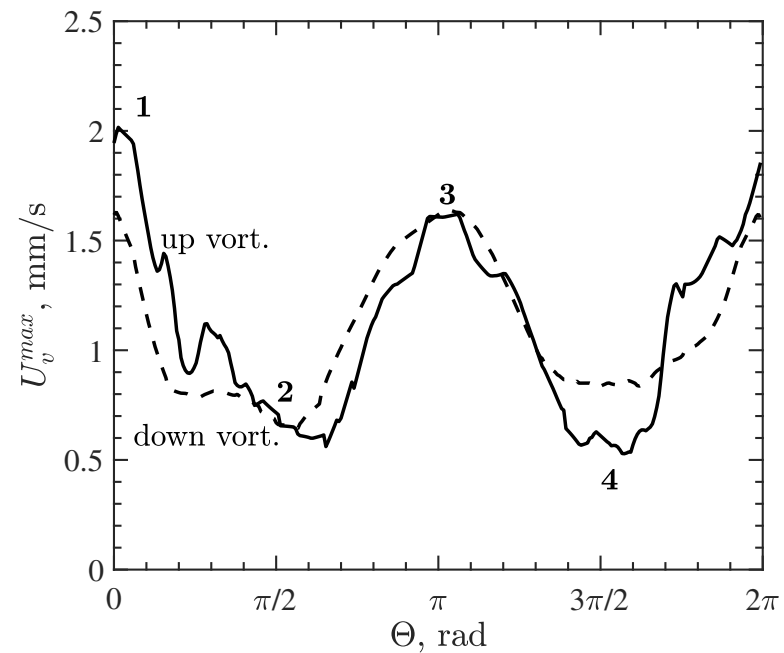

(a)

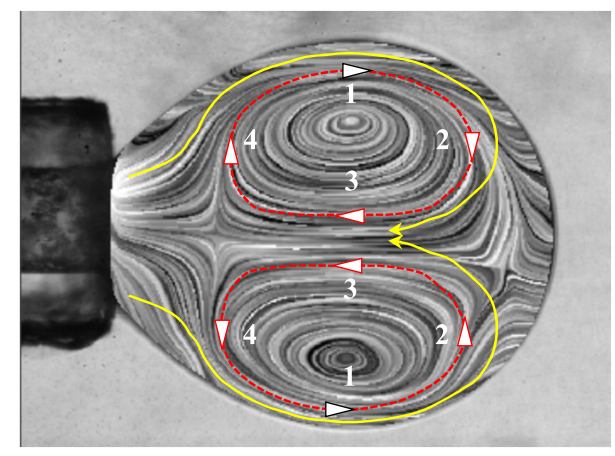

(b)

FIG. 11. Tangential velocities versus polar angle $\Theta$ (a) along the upper and lower vortex streamlines indicated by the red lines (b). The solid and dashed lines correspond to the upper and lower vortices, respectively.

$I_{\omega}$ changes weakly in time with regards to the mean value $I_{\omega} \approx 3 \mathrm{~s}^{-1}$ indicated by the horizontal line inherent to $Q_{c}=$ $0.1 \mathrm{~mL} / \mathrm{min}$. This is observed especially at small flow rates of the dispersed phase, corresponding to $\Omega<0.1$ (Figs. 13a and 13b). In particular, for $Q_{c}=0.1 \mathrm{~mL} / \mathrm{min}$ and $Q_{d}=0.05$ and $0.1 \mathrm{~mL} / \mathrm{min}$, the parameter $I_{\omega}$ weakly differs from the value of $3 \mathrm{~s}^{-1}$. Apparently, the greater the parameter $\Omega$ is, the larger is the decrease in vortex flow intensity with droplet growth (Fig. 13c and 13d). Fig. 14 illustrates the influence of the dispersed and continuous phase viscosity ratio $m$ on the evolution of the vortex flow intensity $I_{\omega}(t)$ for different flow rates $Q_{c}$ and $Q_{d}$ for $m=0.023$ and 0.053 . It is seen that the intensity of pure swirling flow depends only weakly on the flow rate $Q_{d}$ of the dispersed phase and stay within measurement uncertainty. But the intensity of pure swirling flow is determined by the flow rate $Q_{c}$ of the continuous phase. During droplet formation a linear relation between the average vortex rotation rate $I_{\omega}$ and the continuous phase capillary number $C a_{c}=\eta_{c} U_{c} / \sigma$ was observed at different viscosity ratios $m=0.011,0.023$ and 0.053 (see Fig. 15). This is followed 


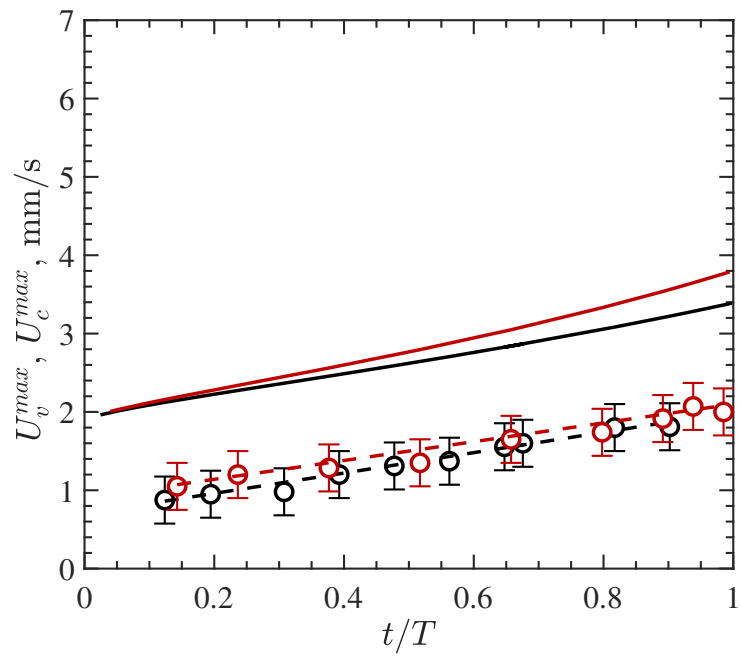

(a)

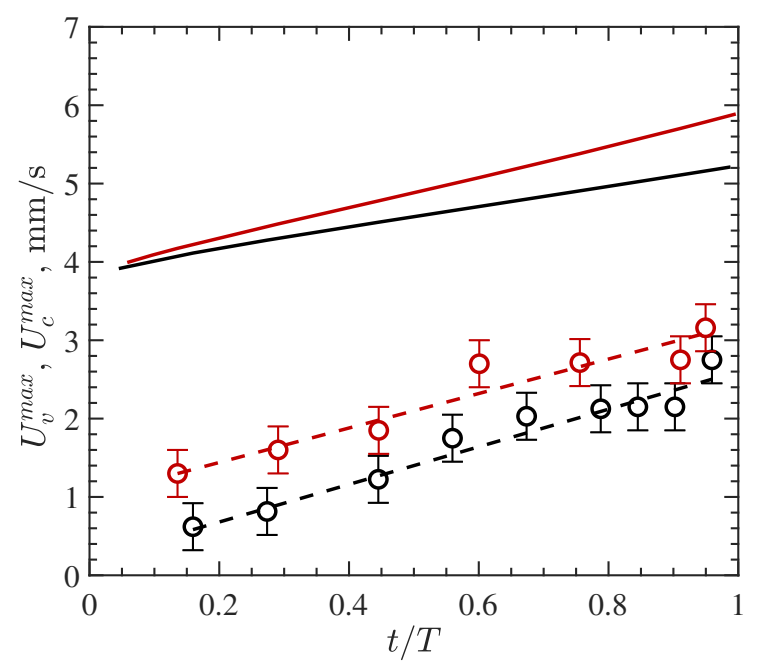

(b)

FIG. 12. Time dependence of $U_{c}^{\max }$ velocity given by Eq. (7) (solid lines) and the measured $U_{v}^{\max }$ velocity in the droplet (circle symbols and dashed lines) for $Q_{c}=0.1$ (a) and $0.2 \mathrm{~mL} / \mathrm{min}$ (b) at $Q_{d}=0.005$ (black) and $0.01 \mathrm{~mL} / \mathrm{min}$ (red).

by the decrease of $I_{\omega}$ with decrease of $m$ (increase of $\eta_{c}$ ) at a given capillary number.

To estimate the angular velocity $\omega_{\text {rort }}(t)$ of the pure swirling flow during droplet growth, we supposed that the linear velocity of the vortex outer face is (i) proportional to the maximum velocity $U_{c}^{\max }$ of the continuous phase (cf. Eq. (7)) and (ii) inversely proportional to the current droplet radius $r_{d}(t)$. Taking into account Eq. (5) one obtain

$$
\omega_{\text {rort }}(t) \sim \frac{U_{c}^{\max }(t)}{r_{d}(t)} \sim \frac{Q_{c}}{r_{d}(t)\left(r_{c}^{2}-r_{d}^{2}(t)\right)} .
$$

This time dependence is plotted in Fig. 16 for $m=0.023$, $Q_{c}=0.1 \mathrm{~mL} / \mathrm{min}$ and three flow rates of the dispersed phase: $Q_{d}=0.005,0.01$ and $0.05 \mathrm{~mL} / \mathrm{min}$. As can be seen from this figure, the angular velocity $\omega_{\text {rort }}(t)$ is very slightly dependent on the flow rate $Q_{d}$ and of the time in the time interval $0.2<t / T<1$ which agrees with the experimental results presented in Figs. 13 and 14. This effect can be explained as follows. Droplet growth is accompanied by an increase in velocity of the continuous phase between the droplet interface and outer capillary. In turn, this leads to an increase in linear velocity along the vortex streamlines (Fig. 12) and, consequently, an increase in vortex angular velocity. On the other hand, the growth of the droplet induces a decrease in the vortex angular velocity due to an increase in the vortex size. From the obtained results, it follows that for $\Omega<0.1$ (see Fig. 13) the reduction of the angular velocity due to the growth of the droplet is balanced by an increase in velocity of the continuum medium. As a result, vortex flow intensity during droplet growth almost does not change.

\section{Neck forming stage}

It was noted above that additional symmetric vortices are formed in the inflow zone of the droplet at the stage of neck formation. They can be seen in Fig. 17a as closed streamlines in the LIC representation at $\widetilde{t}=t / T=0.992$ for the continuous and dispersed phase flow rates $Q_{c}=0.1 \mathrm{~mL} / \mathrm{min}$ and $Q_{d}=0.025 \mathrm{~mL} / \mathrm{min}$, respectively. The angular velocity fields $\omega_{\text {rort }}$ of the pure swirling flow between inflow and circulation zones are presented in Fig. 17b as the colored spots: the red one corresponds to the clockwise rotation while blue color reflects the counterclockwise rotation. It is seen that areas and intensity of the vortices in the inflow zone close to the inner capillary are visibly larger and directions of their rotation are opposite to those of the circulation zone.

Fig. 18 shows the time dependence of the average rotational velocity $I_{\omega}$ of vortices in the circulation (black line) and inflow (red line) zones for $Q_{c}=0.1 \mathrm{~mL} / \mathrm{min}, Q_{d}=0.025 \mathrm{~mL} / \mathrm{min}$ for a dispersed and continuous phase viscosity ratio $m=0.023$. The numbers correspond to the presented flow patterns. It is seen that the neck formation results in appearance of vortex flow in the inflow zone. This is accompanied by a sharp increase in the average rotation velocity $I_{\omega}$ and a simultaneous attenuation of the vortex flow in the circulation zone just before the droplet detachment.

\section{Vortex flow patterns after droplet detachment}

Fig. 19 shows the time evolution of the pure swirling flow intensity $I_{\omega}$ in a droplet right after its detachment. As earlier, the parameter $I_{\omega}$ was estimated from Eqs. (2) and (3). The upper inset indicates the vortex zones in the upper $S_{u p}$ and lower $S_{\text {down }}$ parts of the droplet. It can be seen that directions of their rotation correspond to vortices observed in the inflow zone before the neck rupture (cf. Fig. 17). The flow patterns presented correspond to the numbered time instants. As can be observed, at the initial time (image 1) the vortices are located in front of the ruptured neck and were formed during the preceding stage of the neck formation (see Figs. 17 and 


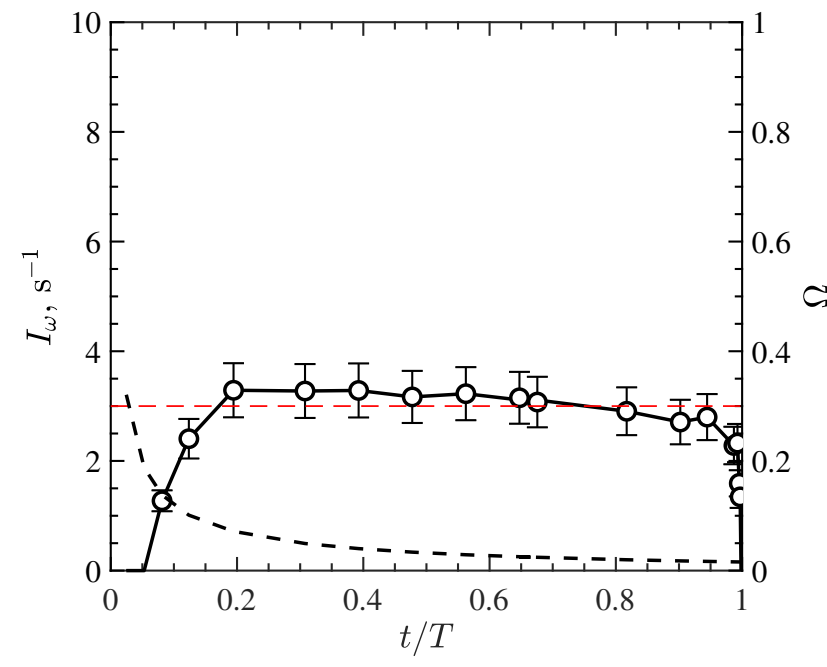

(a)

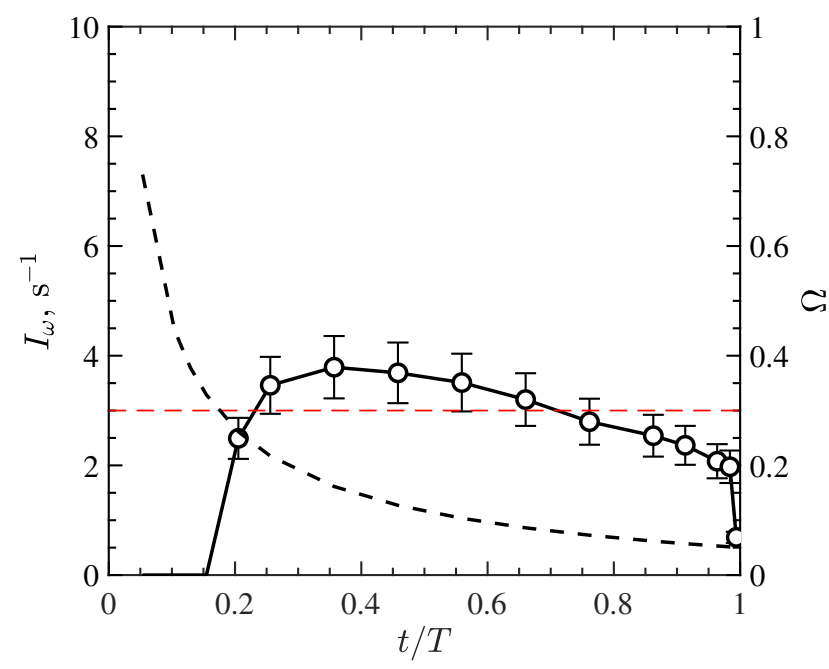

(c)

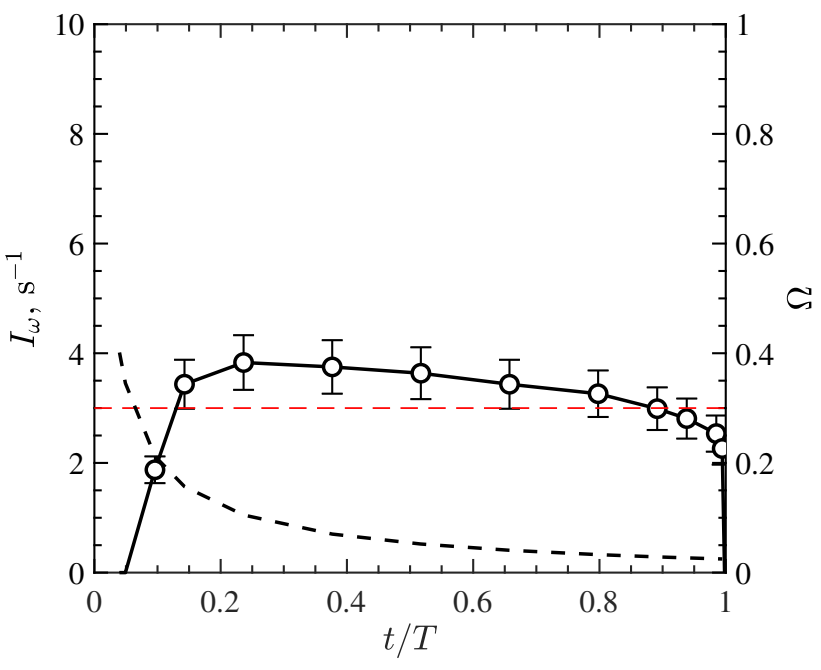

(b)

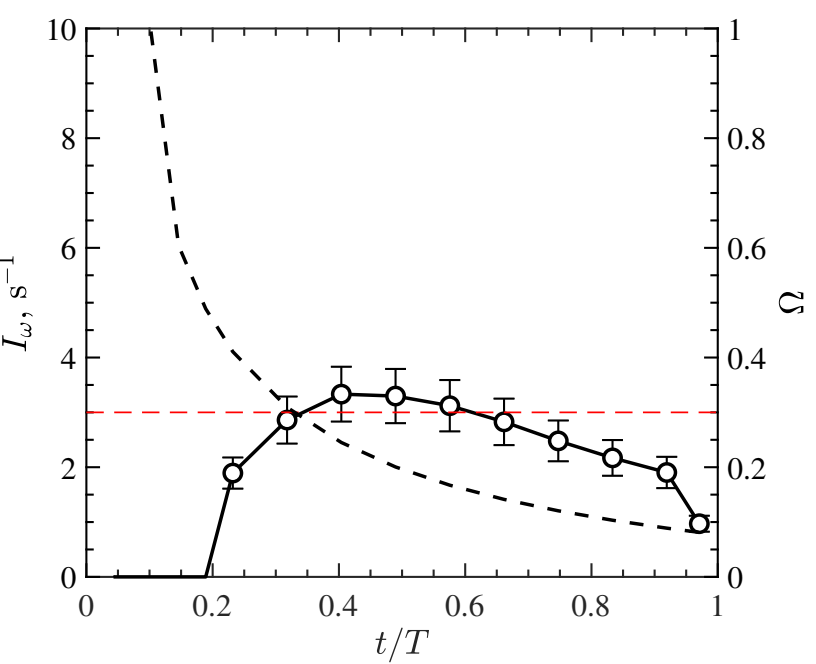

(d)

FIG. 13. Evolution of the vortex flow intensity $I_{\omega}$ (circle symbols, left axis) and $\Omega$ parameter (dashed line, right axis) in a process of droplet formation for $Q_{c}=0.1 \mathrm{~mL} / \mathrm{min}$ and different relative flow rates of the dispersed fluid: $q_{d}=0.05$ (a), 0.1 (b), 0.25 (c) 0.5 (d). Dispersed and continuous phase viscosity ratio $m=0.053$. The dashed red line corresponds to $I_{\omega}=3 \mathrm{~s}^{-1}$.

18). A sharp protrusion remains at the droplet's interface immediately after the droplet detachment. It leads to a jump of capillary pressure caused by high local curvature on the free droplet interface. Under this relatively high pressure, the vortex regions are shifted towards the central part of the droplet (images 2-6). This is accompanied by a sharp decrease in the intensity of the vortex flow with the droplet shape converging to a steady form. This process lasts for one-tenth of a full droplet formation time.

Due to the high speed process, it is appropriate to consider the evolution of the vortex flow intensity $I_{\omega}$ in logarithmic coordinates (see Fig. 20, left axis). It is seen that this process can be divided into three stages. In the first stage, there is an intensive damping of the vortices, which were born during neck formation, including their displacement along the droplet in- terface. A weak growth of $I_{\omega}$ parameter is observed during the second stage while its attenuation can be seen at the third stage. Note that observation of the vortex flow at stage III is limited by the finite microscope field of view (see Fig. 2). Nonetheless, it is reasonable to suggest that outside this region, the vortex angular velocity reaches a steady-state value. It is worth noting that the decay of the vortex intensity during the first stage is linear in logarithmic coordinates which indicates a power dependence on time,

$$
I_{\omega}^{(\mathrm{I})} \sim \widetilde{t}^{-n} .
$$

Fig. 21 shows the variations of index $n$ in Eq. (9) with the dispersed phase flow rate $Q_{d}$ at two flow rates of the continuous medium, $Q_{c}=0.11$ and $0.23 \mathrm{~mL} / \mathrm{min}$, as well as with two 


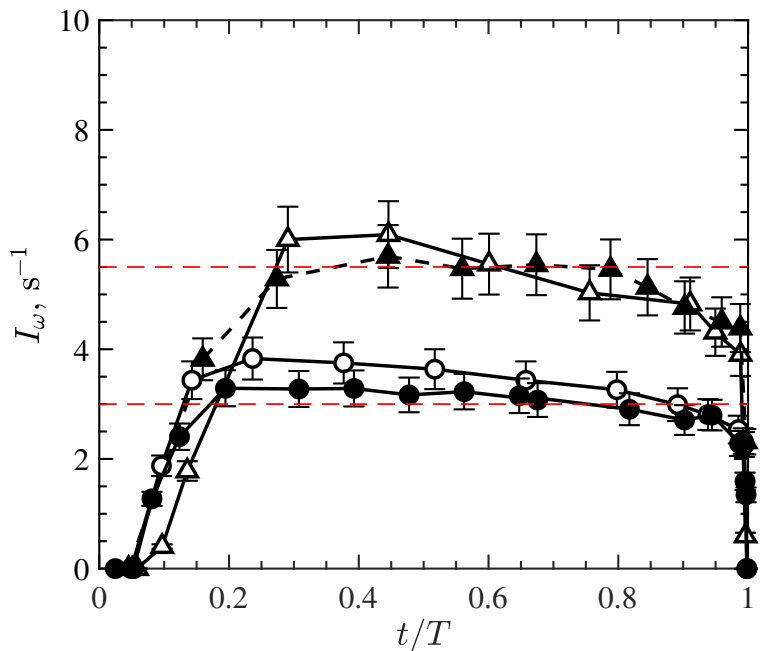

(a)

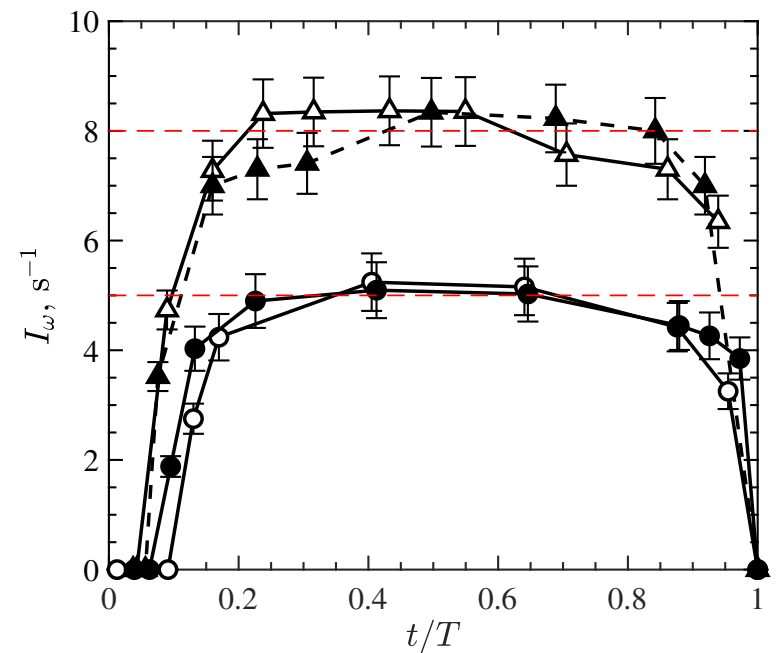

(b)

FIG. 14. Evolution of vortex flow intensity $I_{\omega}$ during droplet formation at (a) $m=0.023$ for $Q_{c}=0.2$ (triangles) and $0.1 \mathrm{~mL} / \mathrm{min}$ (circles) with $Q_{d}=0.01$ (open symbols) and 0.005 (filled symbols) $\mathrm{mL} / \mathrm{min}$ and (b) $m=0.053$ for $Q_{c}=0.23$ (triangles) and $0.114 \mathrm{~mL} / \mathrm{min}$ (circles) with $Q_{d}=0.023$ (open symbols), 0.0114 (filled symbols) $\mathrm{mL} / \mathrm{min}$. The dashed red lines correspond to mean vortex angular velocities at different flow rates of the continuous phase.

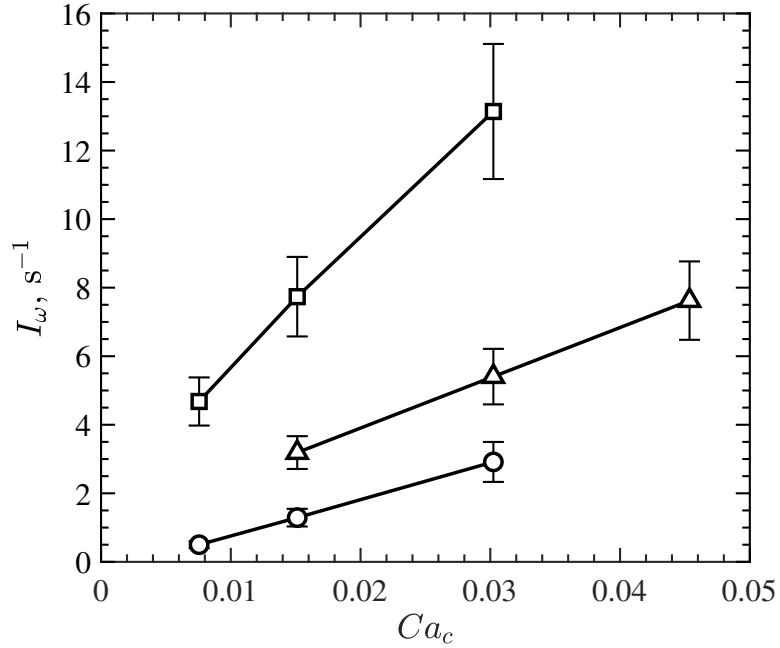

FIG. 15. Dependence of vortex flow intensity $I_{\omega}$ during droplet formation at $\Omega<0.1$ on the capillary number $C a_{c}$ of the continuous phase for different viscosity ratios: $m=0.053$ (squares), 0.023 (triangles), and 0.011 (circles).

values of the droplet relative viscosity $m=0.023$ and 0.053 . It can be concluded that $n$ does not depend on $Q_{d}$ and $Q_{c}$ but increases with $m: n=0.49$ and 0.61 for $m=0.023$ and 0.053 , respectively.

For a better understanding of the observed vortex behavior right after droplet detachment from the inner capillary, we have analyzed the corresponding (i) droplet deformation behavior shown in Fig. 20 (right axis) and (ii) the evolution of the relative velocity $\left(U_{f p}-U_{c}\right) / U_{c}$ of its front point presented in Fig. 22. The droplet deformation was defined as

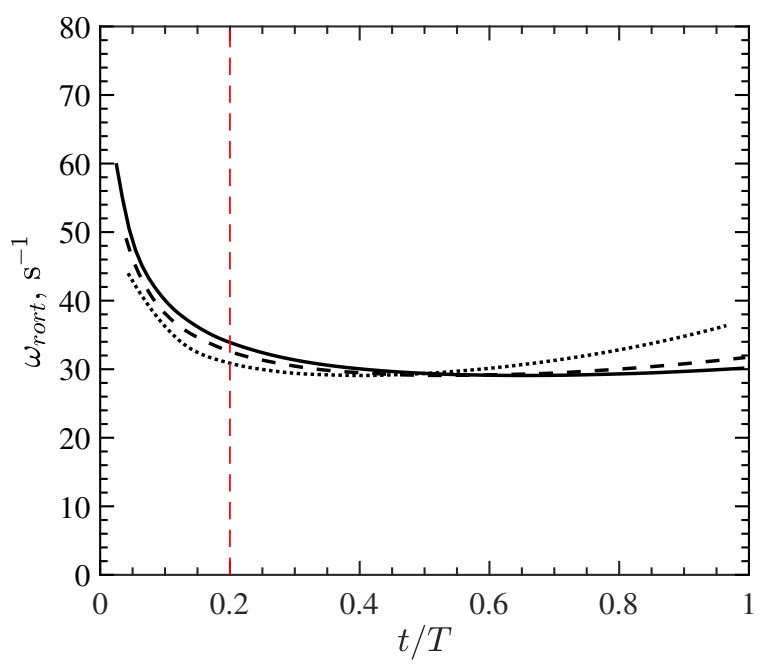

FIG. 16. Dependence of angular velocity $\omega_{\text {rort }}$ on time calculated with Eq. (8) at $Q_{c}=0.1 \mathrm{~mL} / \mathrm{min}$ and $Q_{d}=0.005$ (solid curve), 0.01 (dashed curve) and 0.05 (dotted curve) $\mathrm{mL} / \mathrm{min}$.

its aspect ratio $D=B / A$ of the maximal distances between the droplet interface points in the horizontal $A$ and vertical $B$ directions (see inset in Fig. 20). It can be seen that just after droplet detachment from the inner capillary (stage I) the deformation parameter $D$ increases logarithmically with time, $D \sim \lg (\widetilde{t}-1)$. Then $D$ levels off reaching a steady-state value at the stage III.

On the other hand, since the shape of the interface in the vicinity of the front point is almost unchanged, it could be estimated that velocity $U_{f p}$ corresponds to the droplet velocity as a whole. Fig. 22 shows that at stage I the drop's relative 


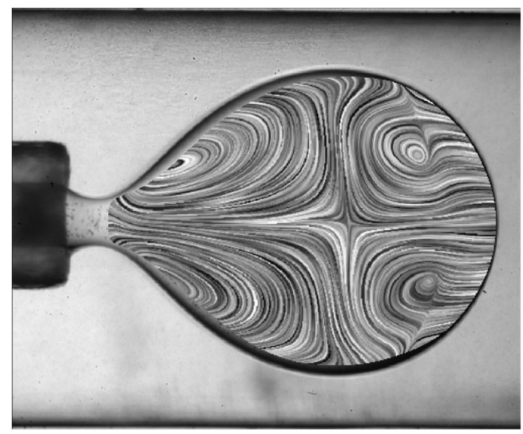

(a)

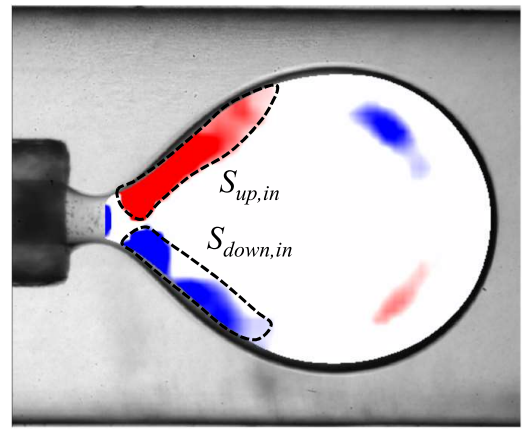

(b)

FIG. 17. LIC representation of streamlines at time $t=0.992 T$ corresponding to the neck formation (a) and angular velocity field $\omega_{\text {rort }}$ of the pure swirling flow (b) for $Q_{c}=0.1 \mathrm{~mL} / \mathrm{min}, Q_{d}=0.025 \mathrm{~mL} / \mathrm{min}$, and $m=0.023$.

velocity is negative due to the fact that its velocity is lower than the average velocity of the continuous medium $U_{c}$ just after droplet detachment. Therefore we can conclude that the change of vortex flow intensity during the first stage is caused by an acceleration of the droplet. This is accompanied by the displacement of the vortices towards the droplet poles under the action of the above mentioned capillary pressure which arising just after detachment (see Fig. 19, image 1). Thus, in spite of high initial intensity of the vortex flow $I_{\omega}$ decreases more than four times for a short time interval (see Fig. 20).

At the beginning of the second stage, the vortices almost reach the droplet poles (see Fig. 19, image 6). At this stage the droplet velocity exceeds the average velocity of the continuous phase. As a consequence, the vortex intensity $I_{\omega}$ is conditioned now by the droplet interaction with the flowing continuous phase. The observed increase in droplet velocity at stage II may be caused by the fact that part of the energy released after the neck rupture increased the droplet's kinetic energy. During the third stage, the droplet reaches a steadystate shape. Its relative velocity and vortex intensity gradually decrease. Additional experiments have shown that small changes in flow rates of the dispersed and continuous fluids do not lead to any qualitative deviations from the revealed peculiarities of the vortex flow behavior in the droplet.

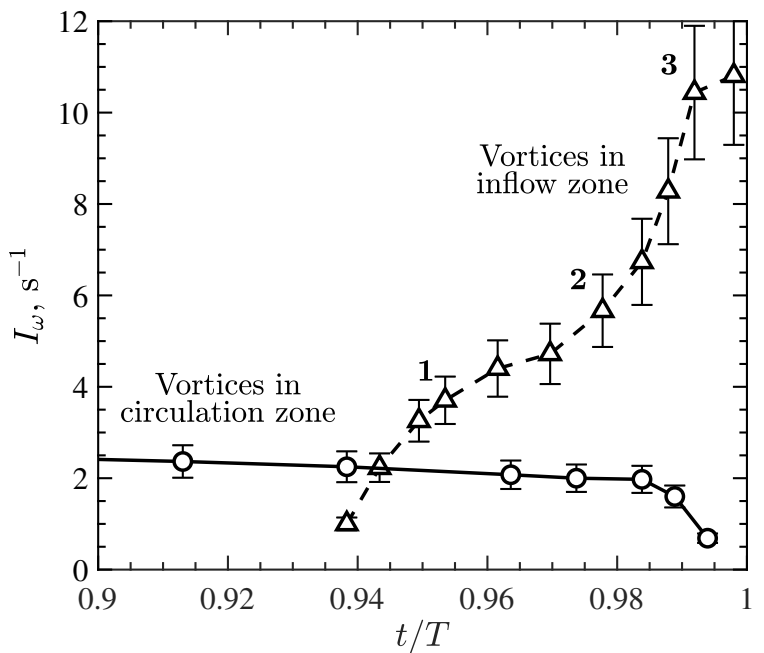

(a)

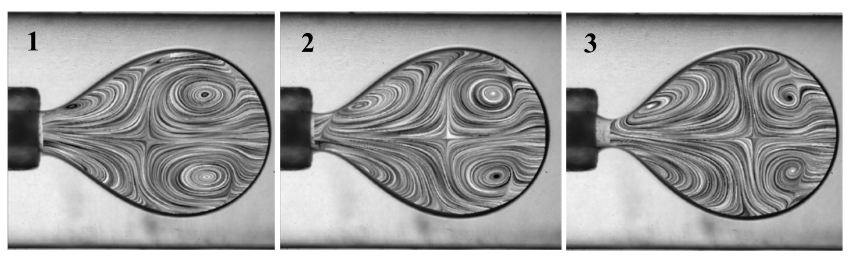

(b)

FIG. 18. Evolution of the vortex flow intensity $I_{\omega}$ in the inflow (circles) and circulation (triangles) zones (a) and flow patterns (b) at the stage of the neck formation at points 1,2 and 3 for $Q_{c}=0.1 \mathrm{~mL} / \mathrm{min}$, $Q_{d}=0.025 \mathrm{~mL} / \mathrm{min}$, and $m=0.023$. The numbers of the flow patterns correspond to the numbers of the triangle symbols.

\section{CONCLUSION}

A comprehensive study of flow pattern development inside a Newtonian droplet has been carried out during its formation in co-flowing geometry in a Newtonian continuous phase in the coaxial capillaries. Each stage as (i) droplet growth, (ii) neck formation, and (iii) droplet detachment were studied sequentially. It was found that during the process of droplet formation the flow pattern represents a superposition of shear and swirling types of flow, whose contribution depends on flow rates of the different phases. The vortex flow intensity parameter $I_{\omega}$, corresponding to average angular velocity of the pure swirling flow and excluding contribution of shear flow, was introduced for a quantitative analysis of experimental data.

In a growing droplet, the flow pattern can be divided into two domains: the inflow zone (near the inner capillary) and the circulation zone (front part of the droplet). The greater the relative flow rate of the dispersed phase, the larger the inflow zone area is. It was found that flow rate $Q_{d}$ of the dispersed phase determines the flow patterns in the inflow zone as well as the droplet growth velocity while the flow rate $Q_{c}$ of the continuous phase determines mainly the intensity and flow structure within the circulation zone. The circulation zone 


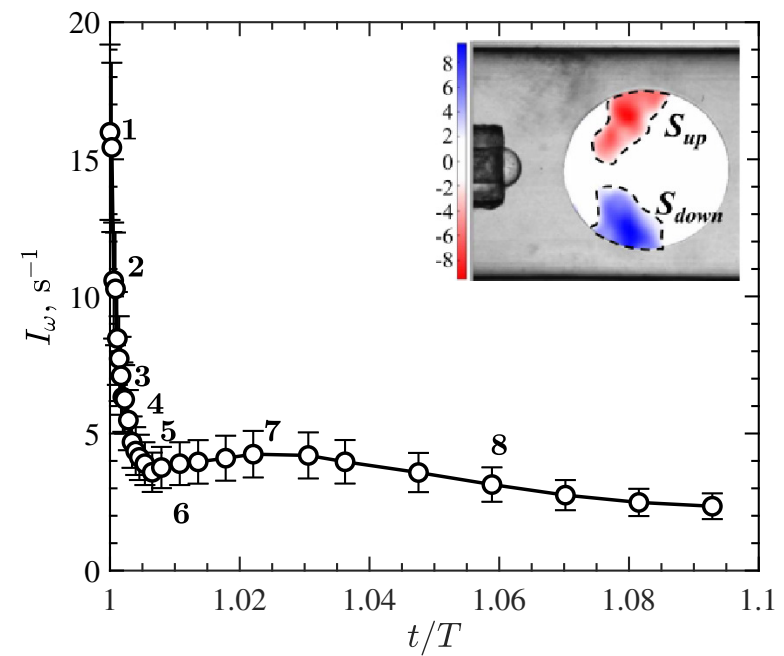

(a)

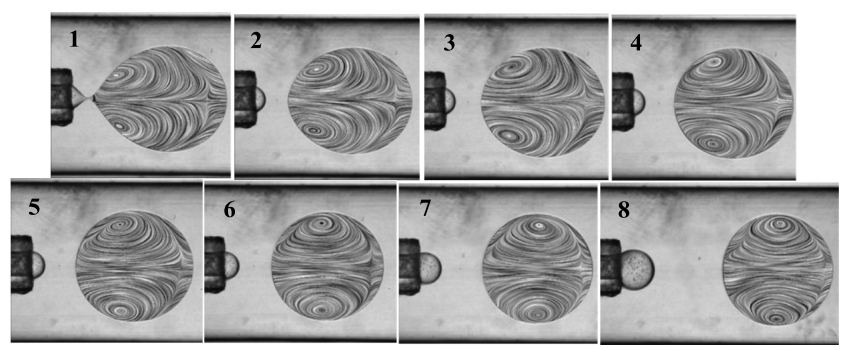

(b)

FIG. 19. Evolution of intensity $I_{\omega}$ (a) and vortex flow patterns (b) immediately after droplet detachment from the inner capillary at $Q_{d}=0.005, Q_{c}=0.1 \mathrm{~mL} / \mathrm{min}$ for a dispersed and continuous phase viscosity ratio $m=0.023$. The numbers of the flow patterns correspond to the numbers of the circle symbols.

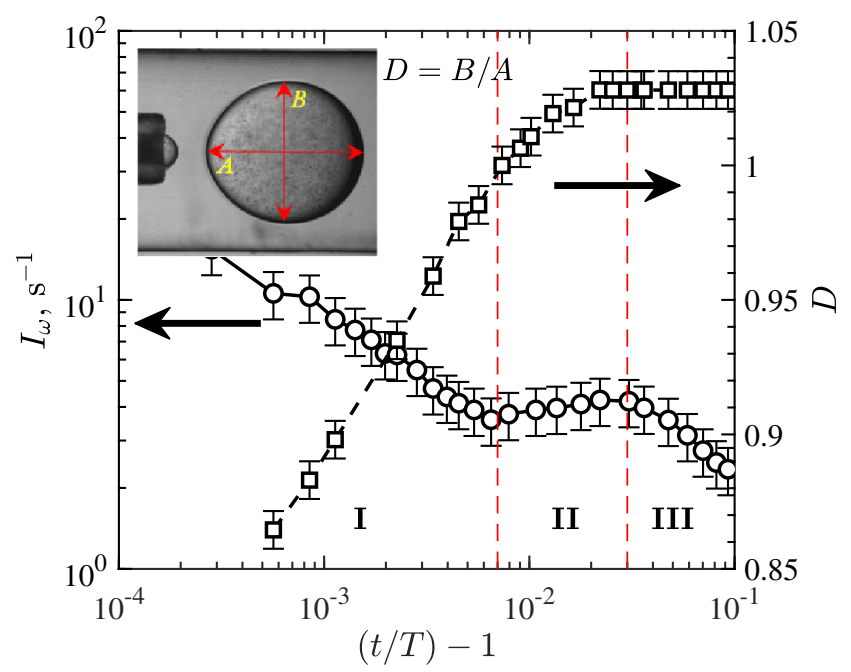

FIG. 20. Evolution of vortex flow intensity $I_{\omega}$ (circles, left axis) and strain parameter $D$ (squares, right axis) just after droplet detachment from the inner capillary at $Q_{d}=0.005 \mathrm{~mL} / \mathrm{min}, Q_{c}=0.1 \mathrm{~mL} / \mathrm{min}$ and $m=0.023$. The vertical dased lines correspond to the boundaries between the three stages of the process.

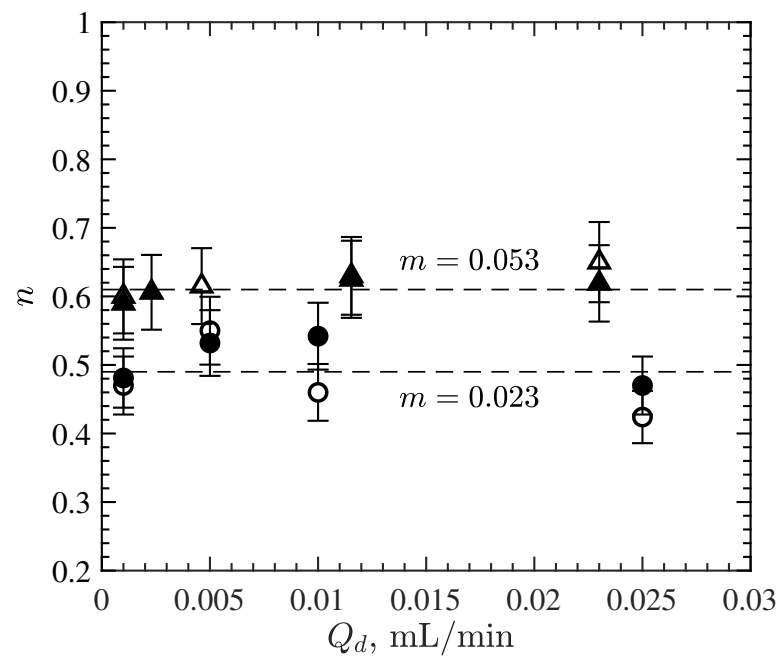

FIG. 21. The dependence of index $n$ on the dispersed phase flow rate $Q_{d}$ for different flow rates of the continuous phase: $Q_{c}=0.11$ (filled symbols) and $0.23 \mathrm{~mL} / \mathrm{min}$ (empty symbols) for two dispersed and continuous phase viscosity ratio : $m=0.023$ (circles) and 0.053 (triangles).

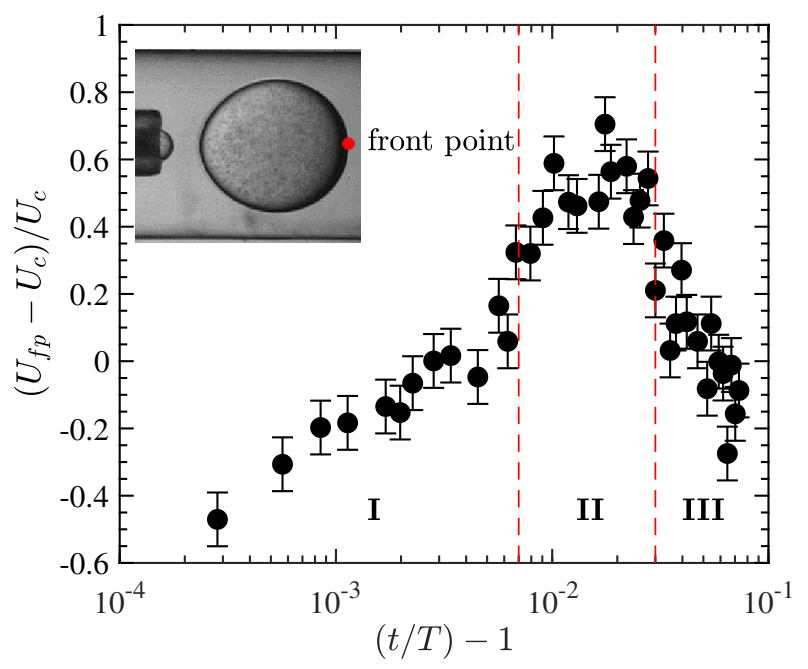

FIG. 22. Evolution of the relative velocity $\left(U_{f p}-U_{c}\right) / U_{c}$ of the frontal point just after droplet detachment from the inner capillary at $Q_{d}=0.005, Q_{c}=0.1 \mathrm{~mL} / \mathrm{min}$ and $m=0.023$. The vertical dashed lines correspond to boundaries between the three stages of the process.

stabilises when the velocity of the continuous phase in the gap between the droplet and outer capillary inner wall dominates significantly over the growth droplet velocity. Otherwise, there are no vortices inside the droplet. In the inflow zone, vortices do not appear before the beginning of the neck formation. Their intensity is almost time-independent. In this stage, an increase in vortex intensity due to the increment in velocity of the continuous phase in a gap between droplet and outer capillary is balanced by its reduction due to growth of vortices. At the same time, vortex flow intensity increases linearly with an increase of the capillary number of continuous 
medium. The increase in the droplet relative viscosity leads to increase in the vortex average angular rotation rate.

At the stage of neck formation, the area and intensity of the vortex flow in the circulation zone shrinks, while a new pair of vortices appears in the inflow zone. The rotation direction of the newly-born vortices is opposite to rotation of the corresponding vortices in the circulation zone. At the time instant of droplet detachment, the circulation zone disappears completely, whereas the vortices in the inflow zone further develop.

\section{ACKNOWLEDGMENTS}

SP and SV are thankful for the financial support of this work by the Russian Foundation for Basic Research (grant no. 18-53-15013) and partly by the State Assignments 00822019-0005 and 0089-2019-0001. CS and DF are grateful to the CNRS for funding through the PRC DroMir project.

The data that support the findings of this study are available from the corresponding author upon reasonable request.

${ }^{1}$ J. W. S. Rayleigh, "On the instability of jets," Proc. London Math. Soc 10, 4-13 (1878).

${ }^{2}$ G. I. Taylor, "The formation of emulsions in definable fields of flow," Proc. R. Soc. London, Ser. A 146, 501-523 (1934).

${ }^{3}$ A. Huebner, S. Sharma, M. Srisa-Art, F. Hollfelder, J. B. Edel, and A. J. deMello, "Microdroplets: A sea of applications?" Lab Chip 8, 1244-1254 (2008).

${ }^{4}$ S. L. Anna, "Droplets and bubbles in microfluidic devices," Annu. Rev. Fluid Mech. 48, 285-309 (2016).

${ }^{5}$ A. T. S. Cerdeira, J. B. L. M. Campos, J. M. Miranda, and J. D. P. Araújo, "Review on microbubbles and microdroplets flowing through microfluidic geometrical elements," Micromachines 11, 201 (2020).

${ }^{6}$ S.-Y. Teh, R. Lin, L.-H. Hung, and A. P. Lee, "Droplet microfluidics," Lab Chip 8, 198-220 (2008).

${ }^{7}$ R. Seemann, M. Brinkmann, T. Pfohl, and S. Herminghaus, "Droplet based microfluidics," Rep. Prog. Phys. 75, 016601 (2012).

${ }^{8}$ N. Convery and N. Gadegaard, "30 years of microfluidics," Micro and Nano Engineering 2, 76-91 (2019).

${ }^{9}$ H. A. Stone, A. D. Stroock, and A. Ajdari, "Engineering flows in small devices: Microfluidics toward a lab-on-a-chip," Ann. Rev. Fluid Mech. 36, 381-411 (2004)

${ }^{10}$ D. Stoecklein and D. D. Carlo, "Nonlinear microfluidics," Analyt. Chem. 91, 296-314 (2019).

${ }^{11}$ M. J. Männel, E. Baysak, and J. Thiele, "Fabrication of microfluidic devices for emulsion formation by microstereolithography," Molecules 26, 2817 (2021)

${ }^{12}$ A. Dewandre, J. Rivero-Rodriguez, Y. Vitry, B. Sobac, and B. Scheid, "Microfluidic droplet generation based on non-embedded co-flow-focusing using 3d printed nozzle," Sci. Rep. 10, 21616 (2020).

${ }^{13}$ Z. Yin, Z. Huang, X. Lin, X. Gao, and F. Bao, "Droplet generation in a flow-focusing microfluidic device with external mechanical vibration," Micromachines 11, 743 (2020).

${ }^{14}$ Y. Geng, S. Ling, J. Huang, and J. Xu, "Multiphase Microfluidics: Fundamentals, Fabrication, and Functions," Small 16, 1906357 (2020).

${ }^{15}$ M. Raffel, C. E. Willert, F. Scarano, C. Kähler, S. T. Wereley, and J. Kompenhans, Particle Image Velocimetry. A Practical Guide (Springer, 2018).

${ }^{16}$ R. Lindken, M. Rossi, S. Große, and J. Westerweel, "Micro-particle image velocimetry (micropiv): recent developments, applications, and guidelines," Lab Chip 9, 2551-2567 (2009).

${ }^{17}$ A. Kislaya, A. Deka, P. Veenstra, D. S. W. Tam, and J. Westerweel, "Psipiv: a novel framework to study unsteady microfluidic flow," Exp. Fluids 61, 1-16 (2020).
${ }^{18}$ T. Nguyen, R. Muyshondt, Y. A. Hassan, and N. K. Anand, "Experimental investigation of cross flow mixing in a randomly packed bed and streamwise vortex characteristics using particle image velocimetry and proper orthogonal decomposition analysis," Phys. Fluids 31, 025101 (2019).

${ }^{19} \mathrm{U}$. Mießner, T. Helmers, R. Lindken, and J. Westerweel, " $\mu$ PIV measurement of the 3D velocity distribution of Taylor droplets moving in a square horizontal channel," Exp. Fluids 61, 125 (2020).

${ }^{20}$ Y. Ding, L. Yu, C. Zhang, H. He, B. Zhang, Q. Liu, D. Yu, and X. Xing, "High-throughput microfluidic particle velocimetry using optical timestretch microscopy," Appl. Phys. Lett. 115, 033702 (2019).

${ }^{21} \mathrm{X}$. Lu, Y. Zhao, and D. J. C. Dennis, "Flow measurements in microporous media using micro-particle image velocimetry," Phys. Rev. Fluids 3, 104202 (2018).

${ }^{22}$ A. Bisten, D. Rudolf, and H. P. Karbstein, "Comparison of flow patterns and droplet deformations of modified sharp-edged and conical orifices during high-pressure homogenisation using $\mu$ PIV," Microfluid. Nanofluid. 22, 57 (2018).

${ }^{23} \mathrm{~F}$. Shen, Y. Li, Z. Liu, and X. Li, "Study of flow behaviors of droplet merging and splitting in microchannels using Micro-PIV measurement," Microfluid. Nanofluid. 21, 66 (2017).

${ }^{24}$ J. R. Burns and C. Ramshaw, "The intensification of rapid reactions in multiphase systems using slug flow in capillaries," Lab Chip 1, 10-15 (2001).

${ }^{25}$ G. Dummann, U. Quittmann, L. Gröschel, D. W. Agar, O. Wörz, and K. Morgenschweis, "The capillary-microreactor: a new reactor concept for the intensification of heat and mass transfer in liquid-liquid reactions," Catal. Today 79-80, 433-439 (2003).

${ }^{26} \mathrm{X}$. Chen, S. Liu, Y. Chen, and S. Wang, "A review on species mixing in droplets using passive and active micromixers," Int. J. Env. Analyt. Chem. 101, 422-432 (2019).

${ }^{27}$ L. Liu, N. Xiang, and Z. Ni, "Droplet-based microreactor for the production of micro/nano-materials," Electrophoresis 41, 833-851 (2020).

${ }^{28}$ W. Zhang, S. Yang, Q. Lin, H. Cheng, and J. Liu, "Microdroplets as Microreactors for Fast Synthesis of Ketoximes and Amides," J. Org. Chem. 84, 851-859 (2019).

${ }^{29}$ Y. Yang, J. Liu, Z. Chen, W. Niu, R. Li, L. Niu, P. Yang, X. Mu, and B. Tang, "A High-Throughput Screening Method for Determining the Optimized Synthesis Conditions of Quinoxaline Derivatives Using Microdroplet Reaction," Front. Chem. 8, 789 (2020).

${ }^{30} \mathrm{C}$. Liu, J. Li, H. Chen, and R. N. Zare, "Scale-up of microdroplet reactions by heated ultrasonic nebulization," Chem. Sci. 10, 9367-9373 (2019).

${ }^{31}$ C. Liu, Y. Li, and B.-F. Liu, "Micromixers and their applications in kinetic analysis of biochemical reaction," Talanta 205, 120136 (2019).

${ }^{32}$ H. Song, D. L. Chen, and R. F. Ismagilov, "Reactions in droplets in microfluidic channels," Angew. Chem. Int. Ed. 45, 7336-7356 (2006).

${ }^{33}$ P. Zhao, H. P. Gunawardena, X. Zhong, R. N. Zare, and H. Chen, "Microdroplet Ultrafast Reactions Speed Antibody Characterization," Anal. Chem. 93, 3997-4005 (2021).

${ }^{34} \mathrm{X}$. Yan, "Emerging microdroplet chemistry for synthesis and analysis," Int. J. Mass Spectrom. 468, 116639 (2021).

${ }^{35}$ J. Wang, Y. Li, X. Wang, J. Wang, H. Tian, P. Zhao, Y. Tian, Y. Gu, L. Wang, and C. Wang, "Droplet microfluidics for the production of microparticles and nanoparticles," Micromachines 8, 22 (2017).

${ }^{36}$ C. Serra, N. Berton, M. B. L. Prat, and G. Hadziioannou, "A predictive approach of the influence of the operating parameters on the size of polymer particles synthesized in a simplified microfluidic system," Langmuir $\mathbf{2 3}$, 7745-7750 (2007).

${ }^{37}$ W. Yu, C. A. Serra, I. U. Khan, M. Er-Rafik, M. Schmutz, I. Kraus, S. Ding, L. Zhang, M. Bouquey, and R. Muller, "Development of an elongationalflow microprocess for the production of size-controlled nanoemulsions: application to the preparation of monodispersed polymer nanoparticles and composite polymeric microparticles," Macromol. React. Eng. 11, 1600025 (2017).

${ }^{38}$ A. M. Klein, L. Mazutis, I. Akartuna, N. Tallapragada, A. Veres, V. Li, L. Peshkin, D. A. Weitz, and M. W. Kirschner, "Droplet barcoding for single-cell transcriptomics applied to embryonic stem cells," Cell 161, 1187-1201 (2015).

${ }^{39}$ E. Brouzes, M. Medkova, N. Savenelli, D. Marran, M. Twardowski, J. B. Hutchison, J. M. Rothberg, D. R. Link, N. Perrimon, and M. L. Samuels, "Droplet microfluidic technology for single-cell high-throughput screening,” Proc. Natl. Acad. Sci. U. S. A. 106, 14195-14200 (2009). 
${ }^{40}$ T. Alkayyali, T. Cameron, B. Haltli, R. Kerr, and A. Ahmadi, "Microfluidic and cross-linking methods for encapsulation of living cells and bacteria - A review," Anal. Chim. Acta 1053, 1-21 (2019).

${ }^{41}$ Y. Gong, N. Fan, X. Yang, B. Peng, and H. Jiang, "New advances in microfluidic flow cytometry," Electrophoresis 40, 1212-1229 (2019).

${ }^{42}$ A. Vembadi, A. Menachery, and M. A. Qasaimeh, "Cell Cytometry: Review and Perspective on Biotechnological Advances," Front. Bioeng. Biotechnol. 7, 147 (2019).

${ }^{43}$ K. Matuła, F. Rivello, and W. T. S. Huck, "Single-Cell Analysis Using Droplet Microfluidics," Adv. Biosys. 4, 1900188 (2020).

${ }^{44}$ X.-D. Zhu, J. Chu, and Y.-H. Wang, "Advances in Microfluidics Applied to Single Cell Operation,” Biotechnol. J. 13, 1700416 (2018).

${ }^{45}$ H. Lu, H. Wang, Y. Liu, M. Wang, J. Hu, and Q. Yang, "Substance transfer behavior controlled by droplet internal circulation,” Chem. Eng. J. 393, 124657 (2020).

${ }^{46}$ M. Oishi, H. Kinoshita, T. Fujii, and M. Oshima, "Simultaneous measurement of internal and surrounding flows of a moving droplet using multicolour confocal micro-particle image velocimetry (micro-piv)," Meas. Sci. Technol. 22, 105401 (2011).

${ }^{47}$ S. Jakiela, P. M. Korczyk, S. Makulska, O. Cybulski, and P. Garstecki, "Discontinuous transition in a laminar fluid flow: A change of flow topology inside a droplet moving in a micron-size channel," Phys. Rev. Lett. 108, 134501 (2012).

${ }^{48}$ Z. Liu, L. Zhang, Y. Pang, X. Wang, and M. Li, "Micro-PIV investigation of the internal flow transitions inside droplets traveling in a rectangular microchannel," Microfluid. Nanofluid. 21, 180 (2017).

${ }^{49}$ S. Ma, J. M. Sherwood, W. T. S. Huck, and S. Balabani, "On the flow topology inside droplets moving in rectangular microchannels," Lab Chip 14, 3611-3620 (2014).

${ }^{50}$ M. Li, Z. Liu, Y. Pang, C. Yan, J. Wang, S. Zhao, and Q. Zhou, "Flow topology and its transformation inside droplets traveling in rectangular microchannels," Phys. Fluids 32, 052009 (2020).

${ }^{51}$ S. S. Rao and H. Wong, "The motion of long drops in rectangular microchannels at low capillary numbers," J. Fluid Mech. 852, 60-104 (2018).

${ }^{52}$ T. Helmers, P. Kemper, J. Thöming, and U. Mießner, "Modeling the Excess Velocity of Low-Viscous Taylor Droplets in Square Microchannels," Fluids 4, 162 (2019).

${ }^{53}$ N. Nivedita, P. Ligrani, and I. Papautsky, "Dean flow dynamics in lowaspect ratio spiral microchannels," Sci Rep 7, 44072 (2017).

${ }^{54}$ D. Malsch, M. Kielpinski, R. Merthan, J. Albert, G. Mayer, J. Köhler, H. Süße, M. Stahl, and T. Henkel, "Piv-analysis of taylor flow in micro channels," Chem. Eng. J. 135, S166-S172 (2008).

${ }^{55}$ Z. Liu, M. Li, Y. Pang, L. Zhang, Y. Ren, and J. Wang, "Flow characteristics inside droplets moving in a curved microchannel with rectangular section,” Phys. Fluids 31, 022004 (2019).

${ }^{56}$ A. Kovalev, A. Yagodnitsyna, and A. Bilsky, "Plug flow of immiscible liquids with low viscosity ratio in serpentine microchannels," Chem. Eng. J. 417, 127933 (2021).

${ }^{57}$ O. Carrier, F. G. Ergin, H.-Z. Li, B. B. Watz, and D. Funfschilling, "Timeresolved mixing and flow-field measurements during droplet formation in a flow-focusing junction,’ J. Micromech. Microeng. 25, 084014 (2015).

${ }^{58}$ W. Lan, S. Li, Y. Wang, and G. Luo, "Cfd simulation of droplet formation in microchannels by a modified level set method," Ind. Eng. Chem. Res. 53, 4913-4921 (2014).

${ }^{59}$ G. Liu, X. Wang, K. Wang, C. P. Tostado, and G. Luo, "Effect of surface wettability on internal velocity profile during droplet formation process in microfluidic devices,” Int. J. Multiphase Flow 80, 188-193 (2016).

${ }^{60}$ E. Roumpea, N. M. Kovalchuk, M. Chinaud, E. Nowak, M. J. Simmons, and P. Angeli, "Experimental studies on droplet formation in a flowfocusing microchannel in the presence of surfactants," Chem. Eng. J. 195, 507-518 (2019).

${ }^{61}$ N. M. Kovalchuk, M. Sagisaka, K. Steponavicius, D. Vigolo, and M. J. H. Simmons, "Drop formation in microfluidic cross-junction: jetting to dripping to jetting transition," Microfluid. and Nanofluid. 23, 103 (2019).

${ }^{62}$ Q.-Q. Xiong, Z. Chen, S.-W. Li, Y.-D. Wang, and J.-H. Xu, "Micro-piv measurement and cfd simulation of flow field and swirling strength during droplet formation process in a coaxial microchannel," Chem. Eng. Sci. 185, 157-167 (2018).

${ }^{63}$ X. Wang, G. Liu, K. Wang, and G. Luo, "Measurement of internal flow field during droplet formation process accompanied with mass transfer,' Microfluid. Nanofluid. 19, 757-766 (2015).

${ }^{64}$ M. Oishi, H. Kinoshita, T. Fujii, and M. Oshima, "Phase-locked confocal micro-piv measurement for $3 \mathrm{~d}$ flow structure of transient droplet formation mechanism in t-shaped microjunction," Meas. Sci. Technol. 29, 115204 (2018).

${ }^{65}$ J.-y. Qian, X.-j. Li, Z.-X. Gao, and Z.-j. Jin, "Mixing Efficiency Analysis on Droplet Formation Process in Microchannels by Numerical Methods," Processes 7, 33 (2019).

${ }^{66}$ W. Lan, S. Li, and G. Luo, "Numerical and experimental investigation of dripping and jetting flow in a coaxial micro-channel," Chem. Eng. Sci. 134, $76-85$ (2015).

${ }^{67}$ C. Deng, H. Wang, W. Huang, and S. Cheng, "Numerical and experimental study of oil-in-water $(\mathrm{o} / \mathrm{w})$ droplet formation in a co-flowing capillary device," Colloids Surf. 533, 1-8 (2017).

${ }^{68}$ W. Thielicke and E. J. Stamhuis, "Pivlab - towards user-friendly, affordable and accurate digital particle image velocimetry in matlab," J. Open Res. Soft. 2, 30 (2014)

${ }^{69}$ T. Dong, W. H. Weheliye, P. Chausset, and P. Angeli, “An experimental study on the dropinterface partial coalescence with surfactants," Phys. fluids 29, 102101 (2017).

${ }^{70}$ S. Ahmadikhamsi, F. Golfier, C. Oltean, E. Lefévre, and S. A. Bahrani, "Impact of surfactant addition on non-newtonian fluid behavior during viscous fingering in hele-shaw cell," Phys. Fluids 32, 012103 (2020).

${ }^{71}$ C. D. Meinhart, S. T. Wereley, and M. H. B. Gray, "Volume illumination for two-dimensional particle image velocimetry," Meas. Sci. Technol. 11, 809-814 (2000).

${ }^{72}$ S. M. Pizer, E. P. Amburn, J. D. Austin, R. Cromartie, A. Geselowitz, T. Greer, B. ter Haar Romeny, J. B. Zimmerman, and K. Zuiderveld, "Adaptive histogram equalization and its variations," Comput. Gr. Image Process. 39, 355-368 (1987).

${ }^{73}$ J. Soria, "An investigation of the near wake of a circular cylinder using a video-based digital cross-correlation particle image velocimetry technique,” Exp. Therm. Fluid Science 12, 221-233 (1996).

${ }^{74}$ R. J. Adrian, "Dynamic ranges of velocity and spatial resolution of particle image velocimetry," Meas. Sci. Technol. 8, 1393-1398 (1997).

${ }^{75} \mathrm{~W}$. Thielicke, The flapping flight of birds, Ph.D. thesis, University of Groningen (2014).

${ }^{76}$ B. Cabral and L. C. Leedom, "Imaging vector fields using line integral convolution," in Proceedings of the 20th Annual Conference on Computer Graphics and Interactive Techniques (Association for Computing Machinery, 1993) p. 263-270.

${ }^{77}$ R. C. Gonzalez and P. Wintz, Digital image processing (Addison-Wesley Publishing Company, Inc., 1987).

${ }^{78}$ Y. Zhang, K. Liu, H. Xian, and X. Du, "A review of methods for vortex identification in hydroturbines," Renew. Sustain. Energy Rev. 81, 1269-1285 (2018).

${ }^{79}$ V. Kolár, "Vortex identification: New requirements and limitations," Int. J. Heat Fluid Flow 28, 638-652 (2007).

${ }^{80}$ J. Jeong and F. Hussain, "On the identification of a vortex," J. Fluid Mech. 285, 69-94 (1995).

${ }^{81}$ C. H. Berdahl and D. S. Thompson, "Eduction of swirling structure using the velocity gradient tensor," AIAA J. 31, 97-103 (1993).

${ }^{82}$ J. Zhou, R. J. Adrian, S. Balachandar, and T. M. Kendall, "Mechanisms for generating coherent packets of hairpin vortices in channel flow," AIAA J. 387, 353-396 (1999).

${ }^{83}$ C. Liu, Y. Wang, Y. Yang, and Z. Duan, "New omega vortex identification method," Sci. China Phys. Mech. Astron. 59, 684711 (2016).

${ }^{84}$ M. S. Chong, A. E. Perry, and B. J. Cantwell, "A general classification of threedimensional flow fields," Phys. Fluids A 2, 765-777 (1990).

${ }^{85}$ Y. Gao and C. Liu, "Rortex and comparison with eigenvalue-based vortex identification criteria," Phys. Fluids 30, 085107 (2018).

${ }^{86}$ C. Liu, Y. Gao, S. Tian, and X. Dong, "Rortex - a new vortex vector definition and vorticity tensor and vector decompositions," Phys. Fluids 30, 035103 (2018).

${ }^{87}$ S. Tian, Y. Gao, X. Dong, and C. Liu, "Definitions of vortex vector and vortex,” J. Fluid Mech. 849, 312-339 (2018). 